\title{
Corela
}

Cognition, représentation, langage

11-1 | 2013

Vol. $11, \mathrm{n}^{\circ} 1$

\section{De l'expérience kinesthésique à la structuration prépositionnelle du schème-image du chemin}

\section{Aurélie BARNABÉ}

\section{CpenEdition}

\section{Journals}

Édition électronique

URL : http://journals.openedition.org/corela/2886

DOI : $10.4000 /$ corela. 2886

ISSN : 1638-573X

Éditeur

Cercle linguistique du Centre et de I'Ouest - CerLICO

Référence électronique

Aurélie BARNABÉ, « De l'expérience kinesthésique à la structuration prépositionnelle du schèmeimage du chemin », Corela [En ligne], 11-1 | 2013, mis en ligne le 20 juin 2013, consulté le 30 avril 2019. URL : http://journals.openedition.org/corela/2886 ; DOI : 10.4000/corela.2886

Ce document a été généré automatiquement le 30 avril 2019

\section{(c) (i) (3) (2)}

Corela - cognition, représentation, langage est mis à disposition selon les termes de la licence Creative Commons Attribution - Pas d'Utilisation Commerciale - Partage dans les Mêmes Conditions 4.0 International. 


\title{
De l'expérience kinesthésique à la structuration prépositionnelle du schème-image du chemin
}

\author{
Aurélie BARNABÉ
}

\section{Introduction}

1 La linguistique cognitive conçoit l'organisation du lexique et des constructions comme les traces de la conceptualisation ${ }^{1}$ de l'expérience du monde par le sujet parlant. Elle considère les structures langagières comme le reflet de structures conceptuelles sousjacentes. Les schèmes-images font partie de ces structures. Le concept « schème-image » provient des recherches empiriques sur les relations spatiales menées par Leonard Talmy $(1978,1983)$ et Ronald W. Langacker $(1976,1987)$ dans les années soixante-dix. En 1980, les schèmes-images ont suscité des questions relatives à leur origine, ce qui a donné lieu aux commentaires philosophiques de Mark Johnson (1987). Les schèmes-images sont construits et abstraits à partir de l'expérience incorporée et socialement située du monde, ce qui leur confère à la fois une assise culturelle et sensori-motrice. Ces schèmes dits préconceptuels (Johnson, 1987:4) correspondent à des structures gestaltiques qui facilitent l'appréhension du monde par le sujet (Ibid. : xix). L'objectif du présent article consiste à analyser la structuration linguistique du schème-image du chemin, alternativement désigné path schema (Johnson, 1987:28) et source-path-goal schema (Langacker, 2000: 55, Feldman, 2006: 144; Johnson, 2007: 141). Le concept même « chemin » a fait l'objet d'études variées en linguistique cognitive, comme le montrent les travaux de Lakoff, 1987a, 1987b; Matsumoto, 1996; Lakoff et Johnson, 1999; Talmy, 2000a, 2000b ; Dan I. Slobin, 1996a, 1997, 2003, 2004 ; Jackendoff, 1983, 2002 ; et Hickmann et Hendriks, 2006, pour ne citer que quelques noms parmi les plus connus. L'expression langagière du chemin (que l'on désignera également par "trajectoire») fait donc aujourd'hui l'objet de nombreuses études. Si bon nombre d'entre elles se réfèrent au schème-image du chemin pour expliciter des faits langagiers particuliers (cf. Boas, 2003), 
il n'est jamais fait mention de la structuration précisément morphosyntaxique de ce schème-image. Par conséquent, le présent article propose de retracer les conditions d'élaboration du schème du chemin en prenant en compte, dans un premier temps, la dimension kinesthésique de son développement. Dans un deuxième temps, des microanalyses d'occurrences issues d'un recueil de 500 occurrences sont proposées pour tenter d'assigner une structuration prépositionnelle à ce schème.

\section{1. "Schèmes », " schémas » et « schèmes-images »}

\subsection{Le corps comme relation au monde}

2 Husserl remarque «J'ai pris conscience d'un moi, sujet de toute constitution en général, qui est dans ses expériences potentielles et actuelles ainsi que dans ses habitus" (Husserl, 1947 : 163). Ces « expériences potentielles » considèrent le déplacement du sujet d'un point de départ dans l'espace vers un point d'arrivée, où le sujet envisage d'être orienté vers ce qu'il n'est pas. Cette expérience sensorimotrice fait partie de nos « modes d'expérience ", vécus et éprouvés à l'intérieur d'un domaine d'action, qui, à un niveau conceptuel, structurent nos modes de perception, notre manière de nous orienter et d'interagir avec d'autres objets, événements ou personnes. Par "modes d'expérience ", nous entendons l'inscription culturelle et sociale de la corporéité humaine dans l'environnement, la "corporéité » désignant la conceptualisation et la figuration du rapport incarné du sujet au monde. Ces modes d'expérience sont culturellement partagés et nous aident à déterminer la nature de la compréhension cohérente de notre " monde " (Johnson, 1987 : 175). Celui-ci est construit et délimité par l'action de l'individu en tant qu'acteur corporel, mais également en tant que sujet représentationnel du traitement de l'information (Gallagher, 2005: 120). Le terme «information» regroupe ici tous les éléments écrits, oraux et les phénomènes liés à l'expérience humaine qui participent à l'élaboration des connaissances du sujet (Col et al., 2010 : §15).

Johnson nomme "schèmes-images $\|^{2}$ ces structures conceptuelles relatives à notre sensibilité. Il les définit comme suit :

Human bodily movement, manipulation of objects, and perceptual interactions involve recurring patterns without which our experience would be chaotic and incomprehensible. I call these "image schemata", because they function primarily as abstract structures of images. (Johnson, 1987 : xix)

3 Schèmes, schémas et schèmes-images sont employés alternativement par les linguistes cognitivistes pour se référer aux schèmes-images, comme le fait Lakoff (Lakoff, 1987a : 416-461). Ces trois désignations signalent également la représentation mentale d'unités lexicales et grammaticales conçues abstraitement sous forme de schémas (Talmy, 2000b : 54). Aussi proposons-nous de situer la notion de schéma en linguistique cognitive par ses antécédents historiques.

\subsection{Antécédents historiques du « schème »}

4 Employé par le neurologue Henry Head, le schéma renvoyait d'abord au schéma corporel (Fortis, 2010 : 19). Le psychologue anglais Bartlett emprunte ce terme à Head, et en retient l'idée qu'une structure passée agit sur la reconnaissance du présent, l'assimilant en quelque sorte, tout en se déformant constamment sous son influence (Ibid.: 19). Le 
psychologue américain Attneave (1957) reprend la notion de schéma: son objectif consiste à déterminer si l'apprentissage ou la reconnaissance de configurations peuvent être facilités par la familiarisation préalable d'une structure dont ces configurations sont les variations.

5 Cette structure, forme génératrice de variations, est ce qu'Attneave appelle "schéma " (Attneave, 1957: 81). Celui-ci présente plusieurs caractéristiques: c'est une forme acquise, construite par extraction d'une tendance centrale, correspondant à une sorte de valeur moyenne des instances auxquelles le sujet a été exposé. Le schéma est donc une structure fluente qui se modifie avec l'expérience (Fortis, 2010:18). Le schéma a été utilisé en neurologie, puis en psychologie, pour rendre compte de phénomènes relatifs à la réélaboration de représentations mnémoniques (Ibid. : 20).

\subsection{Le « schème » aujourd'hui}

6 Le schéma, tel qu'il apparaît dans les recherches menées en linguistique cognitive, semble conserver les caractéristiques principales issues de ses usages initiaux et s'avère très similaire au schème-image que Johnson caractérise comme suit :

Image schemata are more general, abstract, and malleable than rich images; and they have definite parts and structural relations that emerge chiefly at the level of our physical or bodily perception and movement. (Johnson, $1987: 28$ )

7 Schémas et schèmes-images présentent une tendance centrale, en fonction de laquelle des déviations sont reconnues. Le schème renvoie lointainement à Kant. Celui-ci définit les schèmes en termes de processus (Kant, [1835] 2006 : 224), qui selon lui, contribuent à structurer notre expérience. Les schèmes, dont l'association aux schèmes-images semble relativement naturelle, font l'objet de nombreux commentaires: Precisely because image schemas are schematic, and also non-propositional, it is not surprising that it is difficult to define them in a concise and concrete manner (Johnson, 1987: 75). Johnson a défini plusieurs schèmes-images dans un objectif initialement linguistique. Sont répertoriés, dans le Tableau n 1 , les principaux schèmes-images qu'il a explorés (Johnson, $1987: 126$ ) :

Tableau $n^{\circ} 1$ : Schèmes-images identifiés par M. Johnson.

\begin{tabular}{|l|l|l|}
\hline CONTAINER & BALANCE & COMPULSION \\
BLOCKAGE & COUNTERFORCE & RESTRAINT-REMOVAL \\
ENABLEMENT & ATTRACTION & MASS-COUNT \\
PATH & LINK & CENTRE-PERIPHERY \\
CYCLE & NEAR-FAR & SCALE \\
PART-WHOLE & MERGING & SPLITTING \\
FULL-EMPTY & MATCHING & SUPERIMPOSITION \\
ITERATION & CONTACT & PROCESS \\
SURFACE & OBJECT & COLLECTION \\
\hline
\end{tabular}

8 La liste de schèmes qui figurent dans ce tableau n'est pas complète. On note par ailleurs que la liste intégrale des schèmes n'est à ce jour pas connue, en supposant, comme le fait Joseph E. Grady (2005), que tous les schèmes puissent être répertoriés. Johnson fait lui- 
même référence au SCALARITY schema et au ROUGH-SMOOTH schema, dans un ouvrage assez récent (2007). Ces schèmes ont donc été créés récemment (Johnson, 2007 : 143). Johnson livre d'ailleurs une nouvelle définition du schème-image: image schemas that ground meaning in our embodiment and yet are not internal representations of an external reality (Johnson \& Rohrer, 2007: 21):

I can summarize my conception of image schema as: recurrent, stable patterns of sensorimotor experience,

"image"-like, in that they preserve the topological structure of the perceptual whole, operating dynamically in and across time, at once "bodily" and "mental", predicated on interaction with a wider environment, realized as activation patterns (or "contours") in topological neural maps, structures that link sensorimotor experiences to conceptualization and language; and having internal structures that give rise to constrained inferences. (Johnson, $2007: 144$

Image schemas are an important form of conceptual structure in the cognitive semantics literature. The basic idea is that because of our physical experience of being and acting in the world - of perceiving the environment, moving our bodies, exerting and experiencing force, etc. - we form basic conceptual structures which we then use to organize thought across a range of more abstract domains. (Saeed, 1997: 353)

"Image schemas" do indeed constitute a level of meaning prior to the sign.

(Sonesson, $2007: 86$ )

Les schèmes-images font partie de ce que les individus expérimentent quotidiennement. Ces schèmes établissent des expériences renouvelables et des relations relativement fiables entre elles. Les schèmes représentent ce à quoi les organismes sont confrontés, et ce qu'ils expérimentent en tant que "réalité ». Le sujet empirique crée cette réalité, par conséquent, les schèmes manifestent des régularités interprétatives. Mais ces régularités révèlent aussi les contraintes et les limites de ces schémas. Cela implique que notre cognition du réel se manifeste elle-même uniquement là où nos constructions échouent (Korzybski, 1998 : 20-43). Il en résulte que l'expérience de ces schèmes-images se limite à des modèles de connaissance élaborés par des sujets cognitifs, capables de construire pour eux-mêmes et à partir de leur propre expérience.

\section{Le schème-image du chemin}

\subsection{Définitions}

Dans le présent article, nous proposons d'examiner le réinvestissement dans la langue du schème-image relatif au déplacement d'une entitée (in)animée d'un point de départ vers un point d'arrivée. Johnson schématise le schème étudié, désigné schème du chemin (PATH schema) $)^{4}$, comme suit (Johnson, $1987: 28$ ) :

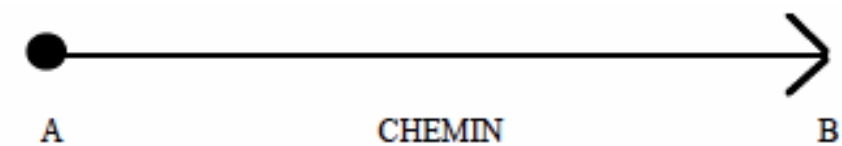

11 La représentation géométrique de ce schème-image renvoie à la réalité physique du chemin dont nous avons l'expérience, par le truchement de notre activité motrice sur ce support spatial. Ce schème-image est constitué de trois sous-schèmes (subschemas) : 
The SOURCE-PATH-GOAL image schema is manifest both in our felt sense of our own bodily movement and also in our tracking of objects through our perceptual field. The SOURCE-PATH-GOAL schema, like all image schemas, is cross-modal, existing kinesthetically, visually, tactilely, and auditorily. (Johnson, 1999: 94)

This FROM-TO schema or PATH schema consists of three elements: a source point A, a terminal point $B$, and a vector tracing a path between them and a relation (specified as a force vector moving from A to B)..$^{5}$ (Johnson, $1987: 28$ )

Le quatrième composant de cette définition est représenté par le sous-schème de la direction (direction subschema), que Lakoff considère vraisemblablement comme inhérent à la définition du schème du chemin :

Structural elements: A SOURCE (starting point), a DESTINATION (end point), a PATH (a sequence of contiguous locations connecting the source and the destination), and a DIRECTION (toward the destination). (Lakoff, 1987a : 275)

On peut supposer que Lakoff a en vue la situation d'un vecteur par rapport à un cadre de référence, toutefois, il ne justifie pas la sélection de la direction, en tant que quatrième sous-schème du schème du chemin. Les travaux sur le schème du chemin font état de sa division en sous-schèmes (Lakoff, 1987a : 285-86) : le sous-schème de la source (source), celui du chemin (path), et le sous-schème du but (goal), qui forment le source-path-goal schema (Langacker, 1991a : 399 ; Lakoff, 1987a : 275 ; Saeed, 1997 : 356 ; Johnson, 2007 : 141). Turner (1996) se réfère même au image schema of movement along a path (Turner, 1996 : 29). La portée du schème du chemin semble rester stable, comme le confirme la définition qu'en donne Johnson, vingt ans après celle qu'il propose dans The Body in The Mind (1987) :

The source-path-goal schema consists of :

1. a starting point,

2. a destination (endpoint), and

3. a path from the starting location to the destination (Johnson, 2007 : 142)

\subsection{Source ontologique du "PATH schema"}

Le schème-image du chemin semble correspondre à la manière qu'a l'individu de structurer ses connaissances, structure que Lakoff désigne par Idealized Cognitive Models ou ICM (Lakoff, 1987a: 68), repris par Andreassen (1997): ICMs are typically quite complex structures, defined by image schemas. (Lakoff, 1987a: 284)

Each ICM has an ontology and a structure. The ontology is the set of elements used in the ICM. The structure consists of the properties of the elements, [which] may be either basic-level concepts - entities, actions, states, properties - or they may be concepts characterized by cognitive models of other types. (Ibid. : 285) [souligné dans le texte]

Lakoff insiste sur la structuration des ICM par les schèmes-images, et précise qu'un ICM peut être, entre autre, propositionnel (propositional ICM) ${ }^{6}$. Il associe la structure-même de l'ICM propositionnel au schème-image du chemin. Selon ses termes, un "scénario " organise la structure de l'ICM :

A scenario consists of [...] an initial state, a sequence of events, and a final state. In other words, the scenario is structured by a SOURCE-PATH-GOAL schema in the time domain, where

- the initial state = the source

- the final state = the destination

- the events = locations on the path 
and the path stretches through time. The scenario is a whole and each of these

elements is a part. (Ibid. : 286)

Le SOURCE-PATH-GOAL schema ainsi décrit s'apparente davantage à un processus. Mais aucune condition d'applicabilité langagière de ce schème n'est indiquée dans cette définition. Nous supposons que cette structure relève d'un ordre conceptuel, comme le précise Lakoff : ICMs are cognitive models, not slices of reality. The "entities" are mental entities, not real things (Ibid. : 285).

\subsection{Premières expériences vécues du schème du chemin}

17 Si la définition du schème du chemin de Lakoff et celles de Johnson sont complémentaires, elles n'ont toutefois pas fait l'objet d'une définition plus large ou plus détaillée du schème-image étudié. Par conséquent, les particularités des trois définitions seront prises en considération dans le présent article. Le schème du chemin, tel que Johnson le définit, ne se restreint pas au déplacement physique d'une entité donnée vers un point d'arrivée, et semble s'étendre à une variété de processus :

This FROM-TO schema is a recurrent structure manifested in a number of seemingly different events, such as: (a) walking from one place to another, (b) throwing a baseball to your sister, (c) punching your brother, (d) giving your mother a present, (e) the melting of ice into water. (Johnson, $1987: 28$ )

La spatialité, en tant que premier repère auquel le sujet est confronté, guide l'élaboration de ses concepts préverbaux (Mandler, 2005 : 139); en cela, elle constitue une contrainte, en tant que base de conceptualisations exécutées par des organismes cognitifs.

Il convient de s'interroger sur l'origine expérientielle du schème du chemin. Selon Mandler (2005: 142), les schèmes-images constituent une description fiable des premières informations auxquelles les enfants assistent. En premier lieu, le jeune individu s'avère être sensible au schème du chemin :

In the first instance, [...] PATH is analysed - that is, an image-schema of an object following any trajectory through space, without regard to the characteristics of the object or the details of the trajectory itself. (Mandler, 2005: 142)

Dans un premier temps, l'attention du jeune enfant se porte exclusivement sur la trajectoire: РATH refers to any object's МотіоN through space, without regard to speed, direction, or shape of either object or path (Mandler, 2010: 26). L'enfant se concentre sur les différents stades du déplacement d'une entité: START PATH refers to the start of motion along a path through space. [...] LINKED PATHS are involved when two objects follow a common path (Ibid.: 27). Mandler présente les premiers sous-schèmes du chemin que l'enfant semble intégrer au cours des premiers mois : PATH, START PATH, END OF PATH, BLOCKED PATH ${ }^{7}$, LINKED PATH, DIRECT PATH (Ibid. : 33).

21 Lorsque l'enfant est attentif à une information spatiale (par exemple le mouvement ou l'arrêt d'une entité), il catégorise et schématise les éléments impliqués dans l'acte de perception. Un processus d'analyse, que Mandler (2005) désigne par perceptual meaning analysis ${ }^{8}$, évalue les renseignements perçus visuellement par l'enfant, et leur attribue une définition simplifiée sous forme de schèmes-images : the format of the descriptions carried out by the perceptual meaning analysis is the image-schema. (Ibid. : 140). Ces schèmes ne sont pas directement accessibles, mais ils permettent de structurer des concepts sous forme d'images ou de mots. 


\subsection{Le chemin et l'acte de mouvement ("motion event")} motion event) de Talmy :

The basic Motion event consists of one object (the Figure) moving or located with respect to another object (the reference object or Ground). It is analyzed as having four components: besides Figure and Ground, there are Path and Motion. (Talmy, $2000 \mathrm{~b}: 25)$

En tant que langue à satellites ${ }^{9}$, la langue anglaise exprime le chemin par une préposition, tandis que le mouvement est encodé par le verbe (Slobin, 2004:26; 2003:161), comme dans l'exemple $(1)^{10}$ :

(1) They crawled into the open space.

Crawl exprime la manière de mouvement, tandis que la préposition into révèle le chemin parcouru par la figure, i.e. they, pour atteindre le fond ${ }^{11}$, i.e. the open space. Into signale le sous-schème du but. Ishibashi et Kopecka (Ishibashi et Kopecka, 2011:2) ont démontré une relative asymétrie des sous-schèmes source / but en faveur du but, à partir des travaux de Talmy (1985), Langacker (1987 : 233), et Slobin (2004).

La relation figure / fond, a fait l'objet de nombreux travaux en sémantique et grammaire cognitives. Herskovits (1986) synthétise le phénomène de localisation qui régit les deux entités :

In the prototypical case where the purpose of the locative expression is to inform the addressee of an object's location, this choice is determined by which of the two objects' location is at issue. That entity is referred to in the subject position of the expression; the entity whose location is taken for granted is referred to in the object position. (Herskovits, 1986: 35)

In summary, there are typical patterns of conceptual movability; [...] The acceptability of certain Figure/Ground choice also varies with the preposition. (Ibid.: 38) [nous soulignons]

Herskovits démontre que l'intention discursive du locuteur, à un niveau conceptuel, oriente nécessairement la disposition syntaxique de la figure et du fond de l'énoncé, la figure apparaissant en position sujet, tandis que le fond occupe le rang de complément, dans un cas prototypique, insiste Herskovits.

Matsumoto (2011) distingue trois éléments, qui, dans l'expression lexicale du chemin, permettent d'identifier des distinctions inter-langagières. Ces trois éléments sont le chemin, la deixis ${ }^{12}$ et la manière de mouvement, soulignés dans le Tableau $n^{\circ} 2$, cidessous :

Tableau $n^{\circ} 2$ : éléments constitutifs du chemin

\begin{tabular}{|c|c|}
\hline figure & $\begin{array}{l}\text { • Entités animées (individus, animaux, collectivités etc.) } \\
\text { 'Entités inanimées (objets) } \\
\text { - Phénomènes produits et / ou dirigés par l'homme : véhicules, voitures, } \\
\text { trains, etc., (balist.) obus, fusée, etc. } \\
\text { ' Matière vivante (bot., végétaux, physiol. anim., muscles, cœur) } \\
\text { - Phénomènes naturels (astron.) terre, soleil, étoiles, (géophys., océanog.) sols, } \\
\text { eaux, (phys.) atomes, électrons). }\end{array}$ \\
\hline
\end{tabular}

Corela, 11-1 | 2013 


\begin{tabular}{|c|c|}
\hline chemin & - Direction du mouvement (toward, up, north, etc.) \\
\hline deixis & $\begin{array}{l}\text { - Acte de référence spatiale dans un espace à trois dimensions, ancré dans un } \\
\text { contexte situationnel et temporel, qui inclut la position spatiale des } \\
\text { participants. }{ }^{13}\end{array}$ \\
\hline manière & $\begin{array}{l}\text { - Outils moteurs nécessaires à l'exécution du mouvement, dynamique des } \\
\text { forces, moyens de transport (animaux, véhicules, trains, avions, etc.). }\end{array}$ \\
\hline contour & • Forme spécifique du chemin (sinueux, en zigzags etc.). \\
\hline étendue & - Durée (il)limitée du mouvement. \\
\hline $\begin{array}{l}\text { franchissement } \\
\text { de frontière }\end{array}$ & ${ }^{\circ}$ Franchissement (ou non) d'une frontière. \\
\hline fond & $\begin{array}{l}\text { • Point de référence (objet, point dans l'espace, entité inanimée etc.), en vertu } \\
\text { duquel l'emplacement et l'orientation de la figure sont repérés. }\end{array}$ \\
\hline
\end{tabular}

Les éléments constitutifs du chemin font écho à ceux définis par Talmy :

Although Path has so far been treated as a simplex constituent, it is better understood as comprising several structurally distinct components :

(1) The Vector: The Vector comprises the basic types of arrival, traversal and departure that a Figural schema can execute with respect to a Ground schema.

(2) The Conformation: The Conformation component of the Path is a geometric complex that relates the fundamental Ground schema within a Motion-aspect formula to the schema for a full Ground object. Each language lexicalizes its own set of such geometric complexes (e.g. from a point which is of the surface of [a volume] = off (of) [a volume]).

(3) The Deictic component of the Path typically has only the two member notions "toward the speaker" and "in a direction other than toward the speaker". (Talmy, 2000b : 53-56; Slobin, 2003a : 13)

Les éléments vector et conformation renvoient conjointement aux différents constituants $\mathrm{du}$ chemin, exposés dans le Tableau $\mathrm{n}^{\circ} 2$. Parmi eux, le franchissement de frontière renvoie à ce que Slobin désigne par boundary-crossing (Slobin, 2003a : 10 ; 1997 : 441).

\section{De l'expérience kinesthésique à la lexicalisation du chemin}

\subsection{Premières formes d'expression du chemin}

Des trois caractéristiques primordiales à l'expression de la trajectoire mentionnées cidessus (i.e. chemin, manière et deixis), celle que le jeune enfant est en mesure de désigner en premier correspond au chemin, d'abord signalé par le geste (Mc Neill, 1992: 162). L'identification de cette première forme d'expression chez le jeune individu soutient la théorie de Vygotsky: The language used in the basic activity of children is a language of gestures and movements (Vygotsky, 1962: 15). Piaget $(1950,1977)$ démontre que les relations topologiques constituent les premières représentations spatiales chez l'enfant. Ces relations correspondent en premier lieu au rapport de proximité, à la fonction de 
séparation, à la structure d'ordre spatial (spatial succession), et à la relation contenant / contenu. Ces relations topologiques correspondent à la signification d'unités prépositionnelles qui permettent au jeune anglophone de lexicaliser le chemin, excluant dans un premier temps la lexicalisation de la manière de mouvement et celle de la deixis (Mandler, 2005 : 145). Selon Hickmann, l'acquisition des prépositions par les anglophones suit un ordre particulier (Hickmann, 2007 : 208).

Ils sont d'abord sensibles aux éléments contenant / contenu, par les prépositions in et out. Ils acquièrent ensuite les relations de support et de contiguïté par on et off. Feldman (Feldman, 2006) désigne l'expérience de support que les enfants indiquent par on, au travers de conceptual schemas plutôt que d'image schemas, considérant cette expérience comme un concept (Feldman, 2006: 144). Les jeunes anglophones intègrent ensuite les notions d'accès (open, close, under) ${ }^{14}$, et de verticalité (up, down, on, under, above, below). En dernier lieu sont assimilées les relations qui impliquent un axe sagittal (behind, in front of), et celles signalant la proximité (next to, between, beside). Les locutions prépositionnelles qui indiquent une projection (in front of, behind) sont acquises plus tard (Allen, $2007: 191$ ) 15 .

\subsection{Distinctions terminologiques inter-langagières}

Par comparaison, des expériences ont porté sur la lexicalisation du chemin par de jeunes Coréens (Hill, 1996; Bowerman, 2007) qui emploient une langue à cadrage verbal ${ }^{16}$. L'expérience a révélé que les enfants décrivent les chemins par des unités verbales. Tomasello (2003) dévoile une étude comparative sur la catégorisation d'objets à localiser par de jeunes anglophones et par des enfants coréens, tous âgés de deux ans. L'expérience confirme les disparités lexicales relatives à l'expression de la localisation ${ }^{17}$. Tandis que les anglophones localisent les entités par les prépositions in et on, les Coréens emploient des verbes pour signaler des relations spatiales équivalentes. (Tomasello, $2003: 63)^{18}$

$\mathrm{Au}$ niveau pré-langagier, la récurrence d'expériences spatiales lexicalisées par des unités linguistiques similaires (cf. prépositions ou verbes) installe une habituation langagière chez le sujet. Par l'emploi d'unités prépositionnelles, le jeune anglophone discerne une entité en mouvement et le point d'arrivée d'une trajectoire donnée, c'est-à-dire la figure et le fond. C'est donc par le truchement de l'unité prépositionnelle que le jeune anglophone manifeste ses premières perceptions langagières du schème-image du chemin.

\subsection{Langues à cadrage satellitaire / langues à cadrage verbal}

Des quatre éléments définissant l'acte de mouvement selon Talmy (Talmy, 2000), à savoir la figure (figure), le fond (ground), le chemin (path) et la manière (manner) de mouvement, c'est la manière de mouvement qui répartit différents groupes de langues, à savoir les langues à cadrage verbal ( $V$-languages) et les langues à cadrage satellitaire (S-languages) ${ }^{19}$. Le sémantisme des verbes appartenant aux langues à cadrage satellitaire favorise l'expression de la manière de mouvement. Stéphanie Pourcel souligne d'ailleurs le MANNER bias qui les caractérise (Pourcel, 2009 : 375).

S-language children have been guided by their native language to pay attention to manner of motion and to construct a set of systematic semantic categories in this domain. [...] In learning the language, the speaker (the child) must surely have 
realized that the language requires him to attend to certain perceptual or conceptual features when he encodes the message. [...] Thus the child begins by "listening (and watching) for understanding", gradually learning to think for speaking (Slobin, 2004: 10) [nous soulignons] une exploration synchronique des outils morphosyntaxiques nécessaires à l'expression langagière du chemin. Nous considérons «l'espace» dans lequel nous évoluons comme une dimension plus riche qu'un simple espace géométrique, en le dotant des propriétés dynamiques, fonctionnelles et intentionnelles que lui confère notre expérience de vie quotidienne (Victorri, 2010 : 2). Dans le présent article, en nous appuyant sur les travaux qui relèvent de la structuration linguistique de la trajectoire, nous nous consacrons à la structuration morphosyntaxique du chemin. 


\subsubsection{Ambivalence sémantique}

41 exemples suivants :

(4) The officer ran into the house where the fugitive was hidden.

(5) The highway runs into Megalopoli and ends into the downtown square.

Ces deux occurrences affichent la même structure syntaxique dans laquelle chaque sujet grammatical a pour verbe run into. Dans l'occurrence (4), cette unité verbale révèle le mouvement effectif de the officer vers le fond que constitue the house. Dans l'exemple (5), run into signale seulement la projection d'un mouvement entre highway et Megalopoli, puisque the highway ne peut révéler un déplacement effectif. Cet énoncé suggère le mouvement « fictif » (fictive motion) de l'entité inanimée the highway (Talmy, 2000a: 99-175). Les figures et fonds de chaque occurrence semblent assigner une acception spatiale spécifique à run into.

\subsubsection{Le schème de la force (FORCE schema)}

Selon Langacker, l'occurrence (4) exemplifie la transmission d'énergie de l'agent ( volitional energy source) vers un patient, i.e energy sink (Langacker, 1991a : 215). L'agent est donc porteur d'une énergie transmise au patient, et la force énergétique incluse dans run into décrit les interactions énergétiques entre figure et fond. Langacker insiste particulièrement sur le déclenchement de la force motrice du chemin, ici représentée par la figure, the officer. Cette approche ne peut s'appliquer à l'exemple (5) de par l'immobilité de la figure, the highway. On observe que la force sur laquelle insiste Langacker renvoie au schème de la force (schema of FORCE), initialement identifié par Johnson (Johnson, 1987 : 46), et amplement commenté par Joanna Podhorodecka (Podhorodecka, 2007: 74). Podhorodecka divise ce schème en d'autres "sous-schèmes", en empruntant les schèmes-images de Johnson. Elle définit la notion de force par l'ATTRACTION schema (Johnson, 1987 : 47), et le compulsion schema (Ibid. : 45), afin de représenter les éléments constitutifs du schème de la force (Podhorodecka, 2007 : 76).

\subsection{La directionnalité : composante inhérente à l'expression langagière du chemin}

Les constructions syntaxiques similaires en (4) et (5) révèlent une divergence d'un point de vue sémantique. L'interprétation de l'association des sujets grammaticaux des deux occurrences à leur structure verbale run into ne renvoie pas à une réalité spatiale identique. Si les exemples mentionnés plus haut reflètent partiellement la lexicalisation du chemin, ils évoquent différentes problématiques. Premièrement, deux constructions syntaxiques similaires incluant le même verbe peuvent renvoyer à une réalité d'ordre spatial qui se présente sous différents aspects. Ces constructions peuvent signaler (i) le mouvement d'une entité donnée ou (ii) son stationnement (stationariness) / sa localisation (Talmy, 2000a: 101). Ce contraste impose un constat: analyser le concept du chemin nécessite que l'attention soit portée sur la direction exprimée par les prépositions. 


\subsection{Méthodologie et structuration verbale du chemin}

Notre recueil de données est composé de 500 occurrences qui exemplifient l'usage de 117 verbes, uniquement employés dans leur acception spatiale ${ }^{21}$. Ces unités verbales regroupent des verbes de mouvement (par ex. run, go) et des verbes de position (par ex. hang, lie). Les données sont présentées dans un tableur qui se compose de cinq rubriques. Un titre a été attribué à chacune d'entre elles.

- La première rubrique (i.e. EXEMPLES) contient l'intégralité des occurrences.

- La deuxième (i.e. VERBES) présente le type de verbe qui correspond à chaque occurrence.

- La troisième (i.e. REGISTRE) signale le genre auquel appartiennent les exemples. Trois genres ont été sélectionnés dans le corpus qui a été choisi pour ces recherches, i.e. Corpus of Contemporary American English (COCA) : la source journalistique (NEWS), le genre romanesque (FICTION), et l'anglais américain parlé (SPOKEN ${ }^{22}$ ).

- La quatrième section est représentée par la source des exemples (i.e. SOURCE), qui indique précisément de quel(s) roman(s), journaux ou émissions de radio les exemples sont extraits.

- La cinquième rubrique indique la date de publication des occurrences (entre 1988 et 2012 exactement).

Tous les exemples commentés dans le présent article sont suivis, entre parenthèses, des trois premières lettres du genre auquel l'occurrence appartient (par ex. "NEW », « FIC », «SPO ») et du numéro précis de l'exemple des données (par ex. NEW, 150). Les 117 unités verbales sont réparties selon dix catégories. Les formes que revêtent ces catégories sont exemplifiées comme suit :

1. Verbes de position : be, face, hang, lie, sit, stand, stay

2. Verbes de mouvement : lead, leave

3. Verbes inchoatifs / BEGINNING type ${ }^{23}$, et terminatif: start, stop

4. Verbes de changement de lieu : come, go, move

5. Verbs signalant une direction: advance, approach, arrive, back, circulate, cross, enter, follow, near, pass, progress, pursue, reach, remove, return, turn

6. Verbes indiquant une manière précise de mouvement: accelerate, amble, cluster, crawl, creep, dart, dash, dawdle, disperse, drag, edge, embark, escape, evade, extend, flit, flow, fly, hop, hurry, inch, lope, march, meander, pace, plod, prance, race, ramble, roll, run, rush, sashay, saunter, scamper, scurry, shuffle, slide, slip, slouch, slow, snake, spread, step, stretch, stroll, swagger, swing, tiptoe, track, trudge, tumble, twirl, waddle, walk, waltz, wander, wiggle, wind, zigzag, zoom

7. Verbes signalant une verticalité ascendante: bounce, climb, escalate, jump, jumpstart, leap, lift, mount, raise, rebound, rise, spring, surface

8. Verbes signalant une verticalité descendante : descend, drop, fall, plunge, shed, slump

9. Verbes indiquant une projection : point, reappear, shine, throw

10. Verbes signalant une perception sensorielle : hear, look, see, smell

Se rapportant aux " catégories ", qui, selon Benvensite, " semblent répondre à un modèle constant ", ce dernier précise que «leurs fonctions n'apparaissent clairement que si on les étudie dans l'exercice du langage et dans la production du discours. » (Benveniste, 1974 : 67). C'est à cette tâche que nous nous employons dans le présent article.

On constate qu'une majorité d'unités verbales (cf. catégorie [6]) révèle une manière précise de mouvement (cf. Slobin, 2003a : 9). Compte tenu de la saillance que représente 
la manière de déplacement (cf. Slobin, 1996a, 2003a, 2003b, 2004 ; Matsumoto, 2011), la distinction des différentes catégories de verbes a contribué à renforcer l'évaluation de la structuration langagière du chemin. Les cas se détournant de la typologie talmienne ne sont pas examinés dans le présent article. Il s'agit des lexicalisations du chemin, exprimées à la fois par le verbe, à l'instar des langues à cadrage verbal, et par les prépositions comme les langues à cadrage satellitaire. Leur construction n'appartient alors pas exclusivement à l'une des deux familles de langues ${ }^{24}$ (Croft, 2001).

Nous appréhendons le chemin comme le déplacement d'une entité le long d'une trajectoire pour atteindre un point de référence. L'exemple (5) cité précédemment implique que la localisation qu'induit la lexicalisation du chemin ne sera pas nécessairement envisagée comme un procès perfectif (Langacker, 1987: 261). Le déplacement d'une entité donnée impose une limite spatiale, ce qui soumet alors l'entité à un changement de localisation, changement et mouvement s'avérant difficiles à distinguer (Barnabé, 2010).

We will not pursue the question of whether change and motion are in fact ultimately distinguishable [...]. (Langacker, 1987: 170)

\subsection{Les satellites}

50 Nous proposons une définition des satellites qui a une incidence directe sur leur considération dans nos données et sur l'expression morphosyntaxique du chemin. Talmy les définit comme suit:

It is the grammatical category of any constituent other than a noun-phrase or prepositional-phrase complement that is in sister relation to the verb root. It relates to the verb root as a dependent to a head. [...] English satellites largely overlap with prepositions - but together, apart, and forth, for example, serve only as satellites, while of, from, and toward serve only as prepositions. (Talmy, 2000b : 102)

51 Talmy insiste sur certaines confusions éventuelles que peut susciter la distinction entre satellites et prépositions. To et over, par exemple, appartiennent aux deux catégories (Ibid. : 106-107) qui, selon Talmy, sont pourtant clairement définies :

Satellites should be well distinguished from prepositions [...] the two forms have quite distinct positional and grammatical characteristics. [...] However, a problem arises in English, which, perhaps alone among Indo-European languages, has come to regularly position satellite and preposition next to each other in a sentence. (Ibid. :106)

L'exemple (6) représente cette juxtaposition de satellite et de préposition, qui peut être une source de confusion :

(6) That guy crawled out of the theater. (NEw, 178)

Dans l'exemple (6), out correspond au satellite tandis que of représente la préposition. Les satellites se distinguent des adpositions dont Claude Hagège propose la définition suivante :

Adpositions (Adps) may be defined as grammatical tools which mark the relationship between two parts of a sentence: one is the element which an adposition governs. [...] English adpositions are prepositions, but other languages have postpositions. [...] Adps are the most frequent type of function marker. [...] Adps constitute, from the semantic and cognitive point of view, a complex set of interrelated meanings. (Hagège, $2010: 6$ ) 

une relation privilégiée (in sister relation to the verb root [Talmy, 2000b : 102]), tandis que les adpositions introduisent des arguments (Grinevald et al., $2011: 4$ ). Les racines lexicales des satellites peuvent être variées : satellites may be verbs, adverbs, preverbs, i.e. grammatical categories that have already been identified in languages (Ibid. : 2). Leur état de satellite est souvent dû à un processus de satellization: satellites constitue seconday systems, in the sense that they are the result of a process of satellization of elements already existing in the language ( Ibid.: 4). Sur le plan sémantique, les satellites font partie des unités lexicales qui structurent l'expression langagière du chemin.

Les unités lexicales, identifiées comme satellites dans une langue donnée, ont une incidence sur les considérations constructionnelles de cette langue. L'emploi de satellites détermine si la langue appartient aux S-framed languages ou aux $V$-framed languages. Talmy, pour classer une langue en tant que S-framed, regroupe par ce terme, satellites et adpositions, ce qui représente une source de confusions puisque ces deux unités (satellite/ adpositions) sont syntaxiquement et lexicalement distinctes. Matsumoto (2011), à cet égard, identifie les langues V-framed de Talmy comme des Head-framed languages, par opposition constructionnelle aux Non-Head framed languages, terme regroupant les satellites et les adpositions, qui correspond aux S-framed languages de Talmy (Ibid. : 3$)^{25}$.

Par souci de précision terminologique, nous distinguons dans le présent article, les adpositions des satellites dans la structuration linguistique du chemin. Nous nous référons aux unités spécifiques que regroupent ces deux termes : nous identifierons d'une part les prépositions (cf. adpositions) et d'autre part les particules, adverbes etc. (cf. satellites). Talmy a identifié un phénomène, constaté en atsugewi ainsi qu'en langue anglaise, qui révèle la combinaison de l'expression du chemin et celle du fond en un même satellite :

(7) After these rites were properly performed, the people turned home in silence. ( FIC, 6)

Home, en tant que satellite au sens talmien, regroupe la préposition et le syntagme prépositionnel to his/her/...home (Talmy, 2000b: 110). Svorou (1994) a identifié des satellites similaires, comme uphill, downhill, uprivers, downriver, qu'il décrit ainsi: Uphill: the trajector is treated as moving in the direction towards the top of the hill (Svorou, 1994: 238). L'emploi des satellites nous renseigne sur la direction spatiale que suggère le chemin, comme dans l'occurrence (8) :

(8) The shark backed off. (sPo, 5)

Les satellites des occurrences (7) et (8) revêtent un sens directionnel. En revanche, il semble problématique d'assigner une acception spatiale à la particule de l'exemple (9) :

(9) The entire force that was with him marched up and approached the city (sPo, 283)

Many languages ${ }^{26}$ have satellites that express aspect. Frequently, these satellites do not indicate purely "the distribution pattern of action through time". This purer form is mixed with or shades off into, indications of manner, quantity, intention, realization and other factors. (Talmy, 2000b:120)

La particule up, dans l'occurrence (9), semble révéler l'accomplissement de l'action, tandis que le sémantisme de march s'avère mettre l'accent sur la manière de se déplacer de the entire force. Comme le souligne Talmy, le satellite peut donner lieu à différentes interprétations. L'occurrence (9) démontre effectivement qu'en dépit du contexte spatial que suggère march, la particule ne désigne pas forcément une précision relative au 
mouvement. Par contre, le sémantisme du verbe nous renseigne immanquablement sur la manière de mouvement.

\section{Les prépositions}

\subsection{L'orientation : une démarche culturelle}

Hagège insiste sur la propriété fonctionnelle des prépositions :

Adpositions can be considered as a fundamental part of speech. The reason is that using lexemes only does not suffice to build a sentence, at least one which is recognized as complete [...]. Certain tools are necessary to link lexemes to one another and to the whole sentence, thereby setting up dependency relationships. (Hagège, 2010: 5)

61 Avant de questionner les restrictions qu'imposent les prépositions pour structurer l'expression linguistique du chemin, l'objet qui nous préoccupe nous oriente vers notre propre discours. C'est pourquoi nous proposons, dans un premier temps, une science de la démarche parallèle à une science de l'objet. La quête sémiotique des unités prépositionnelles dissimule le fondement de leurs types et de leurs limites. Different languages are sensitive to different properties, and they make different "choices» [...]. Languages categorize topological relations strikingly differently. (Bowerman, 2007 : 177-178)

Miller et Johnson-Laird (1976) expliquent que les distinctions organisant l'espace ou sa perception comme front-back et left-right sont universelles, tandis que les points cardinaux (North, South, East, West) représentent les dérivés d'une orientation spatiale que toutes les cultures n'acquièrent pas nécessairement (Givón, 1989)27. L'emploi de prépositions dans la langue anglaise semble être imputable à une représentation du corps culturellement partagée. Selon Heine (1997), le corps ne semble pas être perçu dans une position nécessairement verticale mais dans une posture penchée légèrement en avant (comme lorsque l'on marche). Cette hypothèse implique que la partie du corps head, par exemple, est communément interprétée par le biais des prépositions above et in front of tandis que buttocks s'associe avec below et behind (Heine, 1997 : 57-64). Une autre conséquence de ce profil consiste à conceptualiser la partie supérieure et la partie inférieure du corps en contrepoint, comme le montre le modèle anthropomorphe de la Figure $n^{\circ} 2$ :

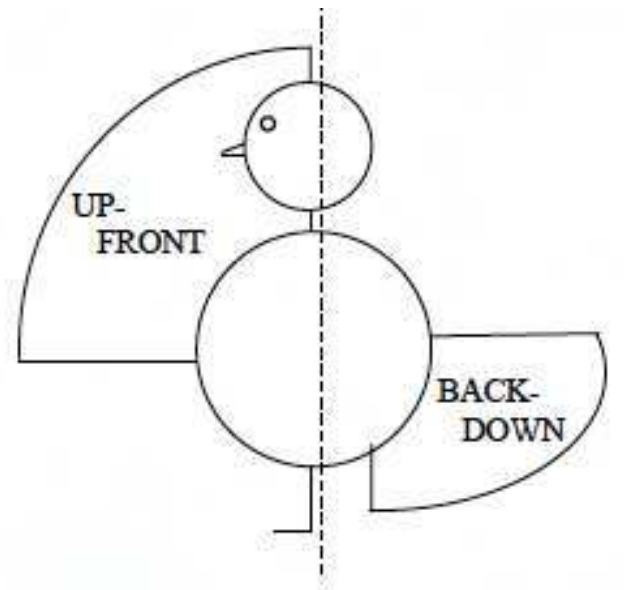




\subsection{Une préposition, un point de vue}

63 Pour localiser deux entités par le biais d'une préposition, le sujet parlant n'évalue pas simplement la distance qui sépare les deux éléments : With projective adpositions [...], a third element is needed, i.e. a point of view (Herskovits, 1986:55). Si les prépositions véhiculées dans la langue anglaise font écho à la structure kinesthésique du modèle anthropomorphe précédemment cité, elles demeurent pour l'essentiel contextualisées, rappelle Svorou (Svorou, 1994: 20). Il identifie leur profil intrinsèque - «les cadres de référence » (references frames) :

The notion which integrates observed behavior with respect to region assignment is the notion of reference frame (RF). This notion is fundamental in many theories of spatial relations with regards to projective relations. [...] Assuming that the region of a Landmark has been partitioned for fine specification of the location of a Trajector, a reference frame assigns values to the sub-regions of the Landmark. ( Ibid. : 21)

Il distingue parmi eux les cadres de référence «inhérents» (inherent $R F$ ), qui correspondent à des prépositions incluses dans des occurrences fournissant suffisamment d'informations spatio-temporelles pour en garantir une bonne réception interprétative par l'interlocuteur. L'occurrence (10) illustre ce type d'emploi prépositionnel :

(10) Trains will enable passengers to go from London to Paris in three hours via the Channel Tunnel. (NEW, 345)

Svorou dissocie ce type d'occurrences des cadres de référence déictiques (deictic RF), dont l'interprétation des prépositions nécessite la présence du sujet parlant dans la scène donnée :

(11) He stepped behind a 10-foot wall of sandbags erected on the right of a cattle ranch. (NEW, 159)

Le mouvement prend en compte la configuration géométrique d'un path-like object, pour reprendre les termes de Langacker (Langacker, 2000 : 158), dont l'orientation esquisse les représentations asymétriques avant-arrière. Ces repères spatiaux sont ceux d'une directionnalité inhérente au mouvement (Svorou, 1994: 184), dont le modèle anthropomorphe demeure la base de perception: The anthropocentric view of directionality constitues the basis for the perception of direction of movement (Ibid. : 25). Les hypothèses de Svorou rejoignent la nécessité du point de vue que défend Herskovits: The fact that motion is understood through perception indicates that there is a point of view which provides a reference frame for specifying the directionality (Herskovits, 1986: 25).

\subsection{Les prépositions, une binarité imposée}

67 C'est précisément à partir du point de vue du locuteur que s'organisent les régions (traduction littérale de region [Svorou, $1994: 12]$ ) qui environnent les entités à localiser. La région n'est pas à confondre avec la "place» aristotélicienne (livre IV, Physique), qualifiée de "neither less nor greater than the thing" (Miller \& Johnson-Laird: 58). Herskovits spécifie une caractéristique relative à ce qu'elle désigne par place:

A place can be thought of as any piece of empty space, as in under the bed is a good place to hide, or - relationally - precisely as that part of space occupied by an object.

[...] Since the only way to describe the place of an object is by using other objects as 
reference, place is linguistically specified by means of a locative expression (Herskovits, 1986: 33). [nous soulignons]

\section{indeterminate penumbra surrounding it. (Miller \& Johnson-Laird, 1976 : 59). Svorou donne un sens très vaste à la région : \\ The knowledge people have about frequently encountered salient entities in everyday life, their size, shape, texture, and function [...] may have several aspects. They know about their component parts, and especially about the part with which they typically interact. Furthermore, they know about the context within which people or other entities interact with particular entities. I propose that all this knowledge may be incorporated into the notion of region of an entity. (Svorou, $1994: 12)$}

La région, au sens où l'entend Svorou, est intrinsèquement liée à l'acte de localisation et de mouvement, à mesure que le locuteur évalue la distance qui sépare les entités à localiser, c'est-à-dire la figure et le fond. Le chemin qui sépare (ou non) la relation figure / fond impose nécessairement une binarité dans le phénomène de localisation :

They did say they did not include KAM Isaiah Israel, which is across the street from Mr. Obama's Hyde Park home. ( $\mathrm{n}^{\circ} 1$, NEW, 81 )

Svorou définit la représentation spatiale de across: Across: the landmark is treated as a stative entity with two boundaries; the trajector is treated as traversing the landmark from one boundary to the other (Svorou, 1994: 237). Across et from sont soumis à deux configurations géométriques distinctes, comme l'indique la Figure $\mathrm{n}^{\circ} 3$ :

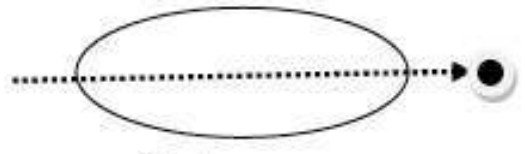

(a) Across

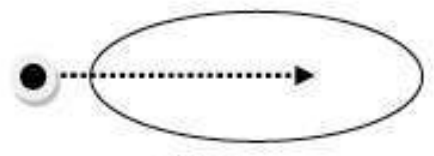

(b) From

Figure $n^{\circ} 3$ : Across et From

\section{La localisation : une marque de subjectivité}

\subsection{Croisement de l'interprétation prépositionnelle et aspectuelle}

Considérons les constructions verbales similaires des exemples (13) et (14) :

(13) The road winds through the granite formations and the many creeks and rivers. (fic, 222)

(14) The road is winding through the mountains and suddenly after many bends in the road, we got to see the Lake Tahoe...it is huge. (spo, 223)

Dans ces deux chemins de localisation, la structure wind through localise la même figure (i.e. road). Seuls la charge aspectuelle et le cotexte ${ }^{28}$ des deux exemples diffèrent. La forme simple de wind, en (13), implique que the road est perçue dans sa globalité par rapport à the granite formations. La construction holistique (pour reprendre les termes de Langacker) de l'exemple (13) rend wind imperfectif; the road ne change pas de position par rapport à 
the granite formations, qui est également immobile. En revanche, la forme be+-ing de l'occurrence (14) semble restreindre l'accès visuel qu'a l'énonciateur de the road. Le cotexte (we got to see the Lake Tahoe...it is huge) démontre que l'énonciateur vient de découvrir l'itinéraire the road (i.e. through the mountains).

On ne peut que confirmer l'effet zooming in de la forme be+-ing en (14), qui fait écho au zooming out qu'évoque Lakoff dans son explicitation des noms pluriels et noms indénombrables (Lakoff, 1987a: 428) qu'il associe aux deux formes aspectuelles examinées. Comparativement à l'exemple (13), la forme be+-ing démontre que l'accès visuel de l'énonciateur à l'entité road est limité. Langacker parle de "construction restrictive $»^{29}$, qui rend wind perfectif dans l'occurrence (14). En effet, the road ne semble pas occuper une position constante et wind, par la forme perfective ${ }^{30}$, paraît signaler un changement dans le temps plus qu'une situation stable (Langacker, 1991b : 96). La forme be+-ing ne fait pas nécessairement référence à une borne du chemin décrit : an imperfective makes no intinsic reference to bounding (Langacker, 2000 : 223).

Le changement perçu et exprimé par la forme perfective relève du point de vue du locuteur, mais pas de la réalité décrite. Seule une connaissance de la situation contextuelle transmise par le cotexte de l'occurrence (14) permet à l'interlocuteur de situer la forme perfective en tant qu'indice du contexte dialogal et de l'intentionalité discursive du sujet: Without the supporting context of the overall gestalt, the remaining profiled states cannot be clearly distinguished and identified (Langacker, $2000: 228$ ).

\subsection{Une localisation asymétrique et subjective}

Localiser une entité par rapport à une autre permet souvent de prendre conscience de la relation asymétrique qu'entretient l'objet localisé avec l'objet localisant (Talmy, 2000a : 316 ; Langacker, 1987).

We may attend to and recognize asymmetrical relations with respect to size, containment, support, orientation, order, direction, distance, motion, or a combination of these. (Svorou, $1994: 8$ )

Chaque instance de localisation implique une construction spatiale subjective (a construal of the spatial arrangement) :

The larger object supports the smaller object, unless balance is involved. If there is no apparent asymmetry between the entity we want to locate and the reference entity, then we impose some kind of asymmetry on it. [...] We take into consideration the location of an observer. (Ibid.: 9) [nous soulignons]

Talmy et Svorou s'accordent à mesurer la saillance des entités localisées et des supports locatifs par la fréquence de leurs emplois respectifs. Cette considération renvoie à la définition du lieu de Vandeloise : " une position dans l'espace chargée d'une valeur aux yeux des participants au discours. [...] Puisqu'un lieu est une position chargée d'une valeur fonctionnelle, leur existence est entièrement subjective » (Vandeloise, 2007b : 348). Les « lieux » auxquels sont attribués les localisations des figures et des fonds révèlent, par leur valeur fonctionnelle, un état subjectif qui correspond aux besoins discursifs des interlocuteurs en interaction, dans une situation donnée. Jackendoff insiste sur la complexité de la configuration des PATHS: The internal structure of a PATH often consists of a path-function and a reference object (Jackendoff, 1993: 163).

La notion de région attenante au PATH de Jackendoff se complexifie dans des occurrences de mouvement. La Figure $n^{\circ} 4$ représente la schématisation du chemin de l'exemple (15) : 
(15)Then you gotta run through the tunnel, then around half the field. (fic, 131)

[Path to ([Place in ([Thing tunnel] $)+[$ Path to ([Place round ([Thing half field] $)$

Figure $n^{\circ} 4$ : chemin de l'occurrence (15)

Le relief interprétatif de cette construction est soumis à la configuration spatiale que Svorou désigne par VIA motion (Svorou, 1994: 27). VIA: the trajector is treated as moving towards the direction of the landmark via (by way of) some secondary reference point (Ibid.: 237). Jackendoff désigne par VIA+place-function le chemin que suggèrent certaines prépositions comme through (Jackendoff, 1993: 166). On note que through est représentée par l'association de to et in dans le schéma de la Figure $n^{\circ} 4$. Le chemin tracé par l'occurrence (15) ne prend son sens comme représentation-description et comme effet que par la «connaissance commune dynamisée» intra-dialogique des locuteurs inscrits dans l'interaction (Hagège, 1998 : 287).

\section{Mouvement et localisation : conceptualisations multiples}

\subsection{Prépositions et sous-schèmes}

Identifier précisément les régions que Langacker qualifie également de search domains (Langacker, 2000: 55), désignés par les prépositions consiste à diviser le schème du chemin en sous-schèmes afin de définir d'éventuelles corrélations entre les unités prépositionnelles et lesdites régions :

The notion of search domain is best understood with respect to one of the cognitively fundamental images schemas proposed by Johnson (1987) and Lakoff (1987), namely source-path-goal. [...] At the clausal level, the path-goal schema is typically manifested in the transmission of energy from an agent to a patient. With prepositions, on the other hand, its usual interpretation pertains to spatial motion. (Langacker, $2000: 55$ )

Selon Langacker, certaines prépositions désignent un transfert d'énergie entre l'agent et le patient (Langacker, 1991a: 209-210). En comparant ces dernières aux prépositions allemandes, il conclut que cette transaction énergétique correspond à l'accusatif et aux sous-schèmes path-goal (par ex. against (gegen), for (für), etc). En revanche, les prépositions qui ne décrivent pas de mouvement ou qui dévoilent un déplacement révélant une "vélocité zéro " ${ }^{31}$ (donc une localisation), équivalent au datif (par ex. on (auf), in (in) etc). Langacker ne spécifiant pas de sous-schèmes correspondant à ce type de prépositions, il mentionne néanmoins que le datif indique soit une absence de mouvement soit un mouvement ayant lieu au sein même d'une localisation. Il semblerait que ces prépositions (cf. in, on) coïncident davantage avec le sous-schème du but puisqu'elles introduisent des syntagmes prépositionnels qui identifient le fond, comme dans l'occurrence (16) :

(16) They have escalated the building of Jewish settlements in occupied territories.

(NEW, $n^{\circ} 351$ )

Bien qu'aucun chemin ne semble ressortir de cet exemple, la préposition in situe, donc localise - selon la grammaire cognitive (Langacker, 1987, 1991, 2000, 2010) - la scène décrite, i.e. they have escalated the building of Jewish settlements. Langacker propose de classer ces deux types de prépositions: les "1-way» prepositions représentent celles qui coïncident avec les sous-schèmes du chemin et du but (path-goal subschemas), et les «2way" prepositions correspondent à celles signalées par le datif (Ibid. : 55). Les prépositions 
qui exemplifient le sous-schème du but se singularisent par la précision de la région qu'elles permettent d'identifier. L'occurrence (17) indique les sous-schèmes du chemin et du but qui désignent un contact entre la figure (i.e. I) et le fond (i.e. the viewing room) :

(17) I walked into the viewing room. (NEW, 139)

Les exemples (18) et (19) font ressortir les sous-schèmes de la source et du chemin :

(18) Then Nessie surfaced out of the lake. (NEW, 401)

(19) One resident said it felt like a tsunami fell from the sky. (FIC, 57)

En ces deux occurrences, les prépositions out of et from mettent en évidence la notion de provenance, commentée dans l'étude du schème du contenant (CONTAINER schema) identifié par Johnson (Johnson, 1987: 21-23). Le CONTAINER schema, amplement commenté par Johnson (Johnson, $2007: 141$ ), correspond à un espace doté de frontières (boundaries) qui mettent en exergue les notions d'intérieur et d'extérieur, i.e. IN-OUT schema (Johnson, 1987 : 34). Il semblerait que les syntagmes prépositionnels out of the lake (cf. (18]) et from the sky (cf. [19]) correspondent aux fonds des deux occurrences. Leur teneur sémantique tend également à ce qu'ils s'associent au sous-schème de la source du chemin. D'un point de vue sémantique, on peut également considérer que out of sous-tend le sous-schème du but dans la mesure où out of peut se comprendre comme « vers un but situé hors d'une source ».

\subsection{Peut-on « définir » une préposition ?}

Par ailleurs, out of, selon Svorou assigne à la relation Nessie/lake une dynamique qui rejoint la force de l'Agonist ${ }^{32}$ de la dynamique des forces de Talmy. La notion de force sous-tend également le schème du contenant: Where there is a container, there can be forces internal to it that are limited and constrained by the boundaries of the container (Johnson, 1987: 35). Les exemples (18) et (19) sont sous-tendus par le sous-schème de la force (FORCE schema), repris par Podhorodecka (Podhorodecka, 2007 : 74). La direction suivie par la figure Nessie, en (18), correspond à celle d'apomacrynsis définie par Svorou (1994). Apomacrynsis: the trajector is physically separated/removed from the landmark by a variety of means, such as breaking, tearing, etc. (Svorou, 1994 : 238).

L'implication des sous-schèmes du source-path-goal semble pour le moins énigmatique avec l'emploi des prépositions with et by, qui affichent un rapport indirect aux sousschèmes du but (Langacker, $2000: 55$ ).

(20) I ran down the street to the bank, then up a wide marble staircase into a magnificent room with a very high ceiling. (NEw, 117)

Les syntagmes prépositionnels introduits par down, to, up et into affichent le chemin que suit la figure I. En revanche, le syntagme prépositionnel introduit par with, en tant que circonstant, se rapporte à magnificient room. Cela démontre que parmi les prépositions incluses dans une occurrence qui retrace un chemin, toutes ne précisent pas nécessairement la configuration spatiale de la trajectoire. Les prépositions with et by expriment davantage une proximité par rapport au fond, mais n'indiquent pas un contact direct comme celui qui caractérise les prépositions sous-tendues par les sous-schèmes du chemin et du but, i.e. path-goal (par ex. to et against).

Eu égard aux figures et aux fonds qui suivent les prépositions along, around, across et through, Svorou ajoute :

Motions, such as ALONG, around, ACROSS and THROUGH, are thus prototypically understood by reference to elongated objects or objects with apparent elongated 
side, spherical objects, concave and transversal objects, or, finally, objects with prominent parallel boundaries, which we may cross. (Svorou, $1994: 28$ ) Svorou: Specifically, movement along the TOP-REGION or BOTTOM-REGION of entities is described as OVER motion or UNDER motion respectively (Svorou, 1994 : 28). Commentant les mêmes prépositions, Jackendoff signale: Many prepositions in English are ambiguous between a pure place-function and To + place-function (Jackendoff, 1983: 163). Under exemplifie l'ambiguïté que soulève Jackendoff. Considérons les exemples suivants :

(23) We put Brigid in the car and then Bug-ette happily came into the house, went under the Christmas tree, and fell asleep. (fic, 347)

(24) Back and forth across the field, they went under the hot summer sun. (fic, 348)

(25) As the ship went under the Bay Bridge, the sailors manned the rails. (new, 349)

(21) Hawthorne would have been astounded by Hester's first glimpse of Dimmesdale : she is wandering through a forest. (FIC, 236) définition de through:

Through: the landmark is treated as a penetrable entity (allowing a passage either because of its loose consistency or because of its shape, e.g. doorway); the trajector is treated as passing through the landmark. (Ibid.: 237) and table) et through (i.e. it) cadrent bien moins avec l'énumération théorique de Svorou sur along, around, across, et through:

(22) Her bright hair fell across the book and table, the ends brushing his wrists and hands. He very much wanted to run his fingers through it. (FIC, 56)

affichent en grande majorité des relations figure / fond qui soutiennent la théorie de Svorou et les deux types de prépositions établis par Langacker. Compte tenu des théories prises en compte pour évaluer nos données, théories qui définissent les prépositions, on conclut que la majorité d'entre elles révèle des régularités interprétatives. Selon le type de chemin dans lesquelles celles-ci sont incluses, et en fonction de leur contexte d'apparition (par exemple, selon leur association à certains types de figures), leur sens ne s'avère pas nécessairement constant. Le passage suivant soulève l'ambiguïté à laquelle peuvent donner lieu des définitions théoriques de prépositions.

\subsection{Le cas de "under"}

a région inférieure (ВоTTOM-REGION) est mise en évidence dans les schémas de la Figure $n$ ${ }^{\circ} 4$, mais de manière bien distincte dans chaque cas : 


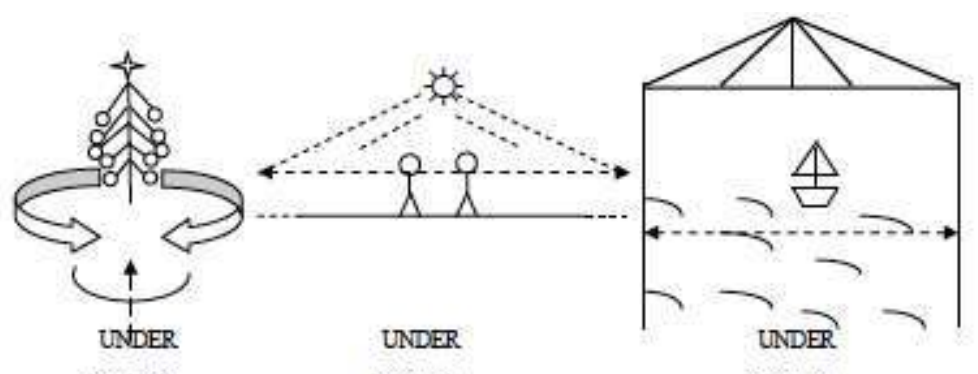

(a) (23)

(b) (24)

(c) (25)

Figure $\pi^{\circ} 4$ : under permet pas nécessairement de se positionner en-dessous du fond (i.e. the Christmas tree [a]). En cette occurrence, under the Christmas tree décrit davantage une relation de proximité. L'interlocuteur est astreint à construire une région (au sens de Svorou) géométriquement approximative sans bornes définies pour situer le syntagme prépositionnel under the Christmas tree, dont les extrémités sont bien plus réduites qu'en (b), où les frontières spatiales sont inexistantes. Le schéma (c) se distingue des deux autres puisque si l'étendue du pont délimite un large espace, défini par under, elle présente indubitablement des limites spatiales qui en circonscrivent la surface (cf. the Bay Bridge). Les trois chemins lexicalisés par went under décrivent donc des configurations spatiales disparates. En (23), le fond (i.e. Christmas tree) représente un point précisément défini dans l'espace. On l'analyse comme suit, à partir de la théorie de Jackendoff (1983), relative au sémantisme des expressions spatiales :

(26)(a) [She] went under the Christmas tree.

$=>\left[_{\text {Path }}\right.$ TO $\left(\left[_{\text {Place }} \text { UNDER }\left(\left[_{\text {Thing }} \text { CHRISTMAS TREE }\right)\right]\right)\right]^{33}$

La précision de ce chemin tranche avec l'espace infini qu'offre the hot summer sun en (24). Jackendoff qualifie ce chemin de route (route) : celle-ci trace un mouvement caractérisé par VIA+place-function :

$(27)\left(b_{1}\right)$ They went under the hot summer sun.

$\Rightarrow\left[_{\text {Path }}\right.$ VIA $\left(\left[_{\text {Place }}\right.\right.$ UNDER $\left(\left[_{\text {Thing }}\right.\right.$ HOT SUMMER SUN $\left.\left.\left.)\right]\right)\right]$

On peut induire une rupture de mouvement ou une localisation temporaire de they, positionnés sous le hot summer sun. La représentation du chemin serait alors légèrement modifiée :

$(28)\left(b_{2}\right)$ They went under the hot summer sun.

$\Rightarrow>\left[_{\text {Path }}\right.$ VIA $\left(\left[_{\text {Place }}\right.\right.$ UNDER $\left[\right.$ Place $I N\left(\left[_{\text {Thing }}\right.\right.$ HOT SUMMER SUN $\left.\left.\left.)\right]\right)\right]$

S'ajoute à la description du chemin la position statique que représente in, qui signale un stationnement ( $c f$. Place IN). Selon Svorou, la préposition under décrit un chemin qui indique une destination :

Descriptions of under motion may be used to specify destinations. (Svorou, 1994 :

28)

under : the landmark is treated as an asymmetrical object with a top-region and a bottom-region, which are assigned by an inherent or a deictic reference frame. The trajector is located at the bottom region of the landmark, and its region is included within the bottom-region of the landmark. (Ibid. : 235)

99 Jackendoff fait l'amalgame de under et through pour représenter under, comme le montre l'exemple (26) :

(29) As the ship went under the Bay Bridge the sailors manned the rails. 
$=>\left[_{\text {Path }}[\right.$ via + inside $=$ through $]\left(\left[_{\text {Place }}\right.\right.$ under $\left(\left[_{\text {Thing }}\right.\right.$ the bay bridge $\left.\left.\left.)\right]\right)\right]$

100
deux prépositions. Les trois exemples mettent en avant le phénomène d'active zone de Langacker, repris par Taylor (2002).

Active zone: If an entity A participates in a situation, often certain parts of A are more intimately involved in the situation than others. These constitute the active zone of A. (Taylor, $2002: 110$ )

Taylor précise qu'il s'avère très délicat de citer un exemple incluant un syntagme prépositionnel sans avoir recours au concept d'active zone. Il cite l'exemple suivant dont le concept d'active zone est, selon lui, complètement absent :

(30) The Earth moves round the Sun. (Taylor, $2002: 111$ )

\subsection{Typologie des prépositions}

Les prépositions non explorées jusqu'ici ont toutes en commun de convoquer une charge constructionnelle plus complexe que les prépositions dites «1-way» et "2-way» (Langacker, 2000). Lorsqu'une entité en mouvement (i.e. la figure) est repérée par rapport à un point de référence (i.e. le fond), le premier élément fonctionne comme la figure psychologique (psychological figure), localisée en fonction de sa distance et de sa géométrie par rapport au fond psychologique (psychological ground). Chaque élément couvre un espace respectif, ce qui impose un partage biparti (bipartite partitioning) de la scène prise en considération (Talmy, 2000a : 313).

(31) Then, the bird flew to the nest and was smiling to the false bird. (fic, 203)

Dans l'occurrence (31), l'entité bird est considérée en fonction de son déplacement vers le fond nest. En revanche, certaines prépositions nécessitent la construction mentale d'un troisième espace (ou région), qui installe un partage triparti (tripartite scene partitioning) de la scène donnée. On doit alors considérer les deux entités à localiser (la figure et le fond) en fonction de ce troisième espace.

(32) As they rolled past the crowd of cheering people, a couple of girls thought a

Mardi Gras parade was nearing. ( $n^{\circ} 1$, spo, 228)

Afin de repérer les entités mentionnées dans l'exemple (32), il incombe à l'observateur de la scène d'identifier un espace couvrant they et une autre région qui entoure crowd. En outre, il lui appartient d'identifier un espace contigu à crowd à travers lequel il puisse situer le déplacement de they. Dès lors, l'interprétation de ce type de localisation implique la combinaison de la figure et $\mathrm{du}$ fond, qui constituent la figure psychologique ( psychological figure) tandis que la région, en arrière-plan (background), constitue le fond psychologique (psychological ground), comme le démontre la figure 5 :

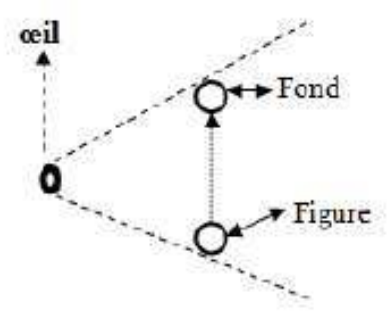

(a) Répartition bipartie (cf. to)

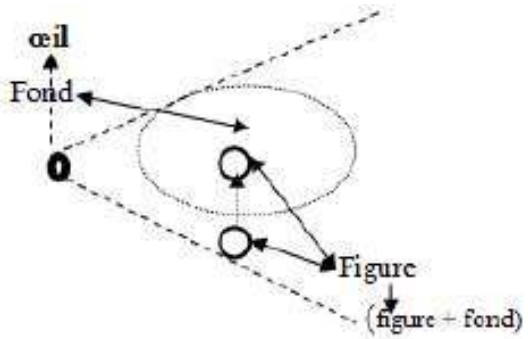

(b) Répartition tripartie (cf. past)

Figure $n^{\circ} 5$ : Répartition bipartie et tripartie de l'espace pour localiser la figure et le fond 
Plusieurs prépositions présentent des distributions triparties (par ex. behind, beside, between, etc.). Le Tableau $\mathrm{n}^{\circ} 3$ présente un classement ternaire des prépositions, dont on précise pour celles dites "1-way» (c'est-à-dire les prépositions qui impliquent le déplacement d'une entité vers un point de référence) à quel(s) sous-schème(s) elles correspondent.

Tableau $n^{\circ} 3$ : Typologie des prépositions

\begin{tabular}{|c|c|c|c|}
\hline \multicolumn{2}{|c|}{ prépositions «1-way» } & prep. «2-way» & prep..$^{34}$ «3-way» \\
\hline $\begin{array}{l}\text { toward } \\
\text { to - after }\end{array}$ & sous-schème but & 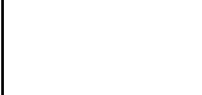 & \\
\hline $\begin{array}{l}\text { out of } \\
\text { from } \\
\text { off } \\
\text { since }\end{array}$ & sous-schème source & on & $\begin{array}{l}\text { Past } \\
\text { behind } \\
\text { beside }\end{array}$ \\
\hline $\begin{array}{l}\text { against } \\
\text { onto/into } \\
\text { behind } \\
\text { with - by } \\
\text { upon } \\
\text { for - down } \\
\text { up - above } \\
\text { below beneath } \\
\text { underneath } \\
\text { until }\end{array}$ & sous-schèmes chemin-but & $\begin{array}{l}\text { at } \\
\text { upon } \\
\text { within } \\
\text { onto } \\
\text { about } \\
\text { around } \\
\text { over } \\
\text { beside } \\
\text { in front of } \\
\text { behind between }\end{array}$ & $\begin{array}{l}\text { in front of } \\
\text { between } \\
\text { among } \\
\text { beyond } \\
\text { round } \\
\text { in front of } \\
\text { around } \\
\text { about } \\
\text { under } \\
\text { over }\end{array}$ \\
\hline $\begin{array}{l}\text { through } \\
\text { across } \\
\text { around } \\
\text { round } \\
\text { along }\end{array}$ & sous-schème chemin & under & down \\
\hline
\end{tabular}

Les prépositions en italique appartiennent à deux classements différents. Cela est dû à leur contexte d'apparition dans les exemples qui les incluent (par exemple, leur rapport avec l'élément "figure ", avec le fond qu'elles introduisent, le cadre topographique qu'elles représentent, etc.). Une majorité d'unités prépositionnelles du tableau s'avèrent appartenir à deux classements distincts. Celles concernées par cette particularité apparaissent soit dans la catégorie "1-way», soit dans la catégorie "2-way» (ces deux classements révèlent peu de compatibilité) et se présentent également dans la catégorie "3-way ", qui illustre leur complexité constructionnelle, mentionnée précédemment (cf. répartition bipartie / tripartie d'une scène donnée). 
107 Cette classification implique que la configuration topographique que suggère une préposition ne peut être décrite de manière stable puisque cette configuration se précise (en partie) en fonction du fond qu'elle introduit et de la figure à laquelle elle est liée. Ce constat renvoie au commentaire d'Aurnague et al. sur les prépositions et autres marqueurs pour localiser une entité par rapport à une autre: "les marqueurs linguistiques sont rarement capables de fournir l'information nécessaire pour déterminer sans erreur la position exacte d'un référent à localiser » (Aurnague et al., 1997 : 71).

\subsection{Accumulation de prépositions}

108 Les langues à satellites ont en commun la facilité d'adjoindre plusieurs prépositions et satellites à un même verbe pour décrire un chemin complexe ${ }^{35}$ (Slobin, 1997, 2003, 2004), comme le souligne I. Ibarretxe-Antuñano citant Slobin :

Narrators in real narratives need not limit a path description to a single verb and its adjuncts [...]; they may present a series of linked paths. (I. Ibarretxe-Antuñano, $2004: 328)$

(33) Finally, the transaction was completed and I ran through and over and around the street construction (NEW, 120)

109 L'occurrence (33) exemplifie les différentes trajectoires qui peuvent s'associer à un même verbe (i.e. run) par les prépositions through, over et around. Such multi-segment paths frequently occur in English descriptions of physical motion [...]. (Slobin, $2004: 17$ )

110 Nous distinguons deux types de chemins complexes. La particularité des premiers se situe au niveau de la structuration de la trajectoire-même :

(34) What did you expect? The rebels to flow down from out of their caves into the towns? (SPO, 221)

111 Le satellite (i.e. la particule down) et les prépositions from et out of sont associés au même verbe, i.e. flow. Ces unités représentent l'enchaînement de directions suivies par the rebels. La complexité de la trajectoire, composée de trois indications de déplacement, est suivie de deux syntagmes nominaux (i.e. their caves et the towns), contrairement à l'exemple (35), dans lequel quatre prépositions introduisent quatre syntagmes nominaux différents :

(35) I ran down the street to the bank, then up a wide marble staircase into a magnificent room with ${ }^{36}$ a very high ceiling. (NEW, 119) ${ }^{37}$

112 Les chemins complexes sont composés de ceux qui présentent un encodage "multiple », i.e. les « chemins complexes $\mathrm{n}^{\circ} 1$ » $(\mathrm{C} \mathrm{c} 1)$, et ceux dont chaque syntagme prépositionnel signale un fond (ground) particulier, i.e. les «chemins complexes $n^{\circ} 2 »(\mathrm{Cc} 2) . \mathrm{Si}$ l'accumulation de prépositions représente une caractéristique des langues à satellites, la récurrence des chemins complexes reste moindre dans la structuration de trajectoires apparaissant dans la langue anglaise (Barnabé, 2012 : 190).

\section{Conclusion}

113 L'objectif de ce travail qui consistait à tenter d'évaluer la structuration syntaxique du schème-image du chemin ne pouvait se départir d'une quête épistémologique du conceptmême de "schème-image ». L'expérience vécue du PATH schema fait nécessairement ressortir la spatialité comme guide mais surtout comme contrainte et base de conceptualisations multiples de l'organisme cognitif. Mandler (2005) précise que le schème du chemin constitue la première structure conceptuelle vécue par le jeune enfant, 
structure de fait appréhendée dès la naissance par l'individu. Des trois caractéristiques primordiales à l'expression de la trajectoire (i.e. chemin, manière et deixis), le chemin représente le sous-schème que le jeune anglophone désigne prioritairement par le truchement de prépositions et/ou particules. Dans la logique des langues à cadrage satellitaire, l'analyse d'un recueil de 500 occurrences a clairement démontré l'emploi massif de verbes spécifiant une manière de mouvement relativement précise pour structurer le chemin tel qu'il a été défini par Mark Johnson en 1987 (Johnson, $1987: 28$ ) et plus récemment en 2007 (Johnson, 2007 : 142). Si les prépositions associées aux unités verbales permettent d'approfondir la précision topographique attribuée aux trajectoires véhiculées dans la chaîne parlée, la quête sémiotique des unités prépositionnelles nous soumet également à notre propre discours. Les exemples commentés dans le présent article démontrent le manque de fiabilité révélé par l'acte strictement définitoire des prépositions. Énoncer une trajectoire et donner une existence signifiée au schème-image du chemin semble, en chaque instance linguistique, attribuer des critères conceptuels et sémantiques ajustables aux prépositions en fonction de l'intention illocutoire de l'énonciateur et de la situation contextuelle dans laquelle il s'insert, ce qui paraît nous détourner d'une thèse "encodagiste ». Les énoncés exemplifiant notre argument par l'emploi de under (cf. [23], [24] et [25]) dénoncent la stabilité de paramètres sémantiques stables pour tenter d'attribuer une valeur constante aux prépositions, à partir des régularités interprétatives qu'elles dévoilent. Les phénomènes de mouvement et de localisation semblent sous-tendre de multiples conceptualisations pour structurer le schème-image du chemin, dont nous venons de voir que la combinaison prépositionnelle n'est qu'un aspect.

\section{BIBLIOGRAPHIE}

ALLEN, S. E. M. 2007. « Interacting pragmatic influences on children's argument realization ». In M. Bowerman et P. Brown. Crosslinguistic Perspective On Argument Structure, 191-213. New York, London: Lawrence Erlbaum Associates.

ANDERSEN, E. 1978. « Lexical universals of body-part terminology ». In J.H. Greenberg. Universals of Human Language. Stanford: Stanford University Press.

ANDREASSEN, L. 1997. «Introduction to Cognitive Models ». Center for Semiotics. Disponible sur : http://www.scribd.com/doc/6015473/George-Lakoff-Cognitive-Models

ARISTOTE. 1933 [1991]. Physique, Tome 1. Livres I-IV. Paris: VRIN.

ATTNEAVE, F. 1957. « Journal of Experimental Psychology ». In American Journal of Psychology, 68: 69-82.

AURNAGUE, M. L. Vieu et A. Borillo. 1997. «Représentation formelle des concepts spatiaux dans la langue ». In The Categorization of Spatial Entities in Language and Cognition, vol. 20, Human Cognitive Processing.

BARNABÉ, A. 2010. « Change conceptualized through motion verbs ». Communication, conférence internationale de la Societas Linguistica Europeae (SLE), Vilnius, septembre 2010. 
BARNABÉ, A. 2011. Définition du SCHÈME-IMAGE DU CHEMIN (path schema) dans le glossaire de l'Association Française de Linguistique Cognitive, (c) AFLiCo.

BARNABÉ, A. 2012. «Corps, perception, déplacements : de l'expérience kinesthésique à la cognition linguistique. Étude du schème du chemin en grammaire et sémantique anglaises et statut de ce schème en linguistique cognitive ». Thèse de doctorat sous la direction de M. J.-R. Lapaire, Université de Bordeaux III.

BARTLETT, F. 1932 [1997]. « Part I: Experimental studies ». In F. Bartlett. Remembering. Cambridge: Cambridge university press.

BENVENISTE, E. 1974. Problèmes de linguistique générale, 2. Paris: Gallimard.

BOAS, H. 2003. A Constructional Approach to Resultatives. Stanford, CA: CSLI Publications.

BOWERMAN, M. 2007. « Containment, support, and beyond: Constructing topological spatial categories in first language acquisition ». In M. Aurnague, M. Hickmann et L. Vieu (eds.), The categorization of spatial entities in language and cognition, 177-203. Amsterdam: John Benjamins.

CHOUINARD, P. 1997. « Speaking of motion: How do children acquiring their first language learn to properly express motion events? ». Thèse de doctorat sous la direction de Dan I. Slobin, Université de Berkeley.

COL, G., J. Aptekman S. Girault et B. Victorri. 2010. « Compositionnalité gestaltiste et construction du sens par instructions dynamiques ». In P. Gréa et G. Désagulier (eds.). Cognitextes 5, Numéro spécial AFLiCo 3. Grammaires en Construction(s). Disponible sur : http://cognitextes.revues.org/372 CROFT, W. 1991. Syntactic Categories and Grammatical Relations: The Cognitive Organization of Information. Chicago: University of Chicago Press.

CROFT, W. 2001. Radical Construction Grammar: Syntactic Theory in Typological Perspective. Oxford: Oxford University Press.

DIXON, R.M.W. 1991. A Semantic Approach to English Grammar. New York: Oxford University Press.

FAUCONNIER, G. 1994. Mental Spaces: Aspects of Meaning Construction In Natural Language.

Cambridge : Cambridge University Press.

FAUCONNIER, G. et M. Turner. 2002. The Way We Think. Conceptual Blending and the Mind's Hidden Complexities. New York: Basic Books.

FAUVELLE, E. 2009. « Les métaphores spatiales, supports des représentations de l'acte d'écrire et aide à l'appropriation ». In M.J. Berchoud (ed.). Les Mots de l'Espace : Entre Expression et Appropriation : Contributions à une Coordination des Points de Vue Autour des Sciences du Langage, 77-86. Paris: L'Harmattan.

FELDMAN, J. A. 2006. From Molecule to Metaphor: A Neural Theory of Language. Cambridge, Mass.: MIT Press.

FILLMORE. 1985. « Frames and the Semantics of Understanding ». Quaderni di Semantica 6, no. 2 : 222-53.

FORTIS, J.-M. 2010. « De l'hypothèse de Sapir-Whorf au prototype : sources et genèse de la théorie d'Eleanor Rosch. ». Revue en ligne Corela, 8(2). Disponible sur : http://corela.revues.org/1243

GALLAGHER. S. 2005. How The Body Shapes The Mind. Oxford/New York: Oxford University Press.

GIVÓN, T. 1989. Mind, Code and Context: Essays in Pragmatics. Hillsdale, New Jersey: Lawrence Erlbaum Associates Publishers. 
GRADY, J. E. 2005. «Image schemas and perception: Refining a definition ». In B. Hampe (ed.). From Perception to Meaning: Image Schemas in Cognitive Linguistics, 35-55. Berlin: Mouton de Gruyter. GRINEVALD, C., C. Imbert et A. Söres, 2011. « About Path 'Satellites': Elements for a multi-layered definition of 'Satellites' « . Communication, Atelier « Trajectoire »: AFLiCo n 4, Lyon 2011.

HAGEGE, C. 1998a. L'Homme de Paroles : Contribution Linguistique aux sciences humaines. Paris: Fayard. HAGEGE, C. 2010. Adpositions. Oxford/New York: Oxford University Press.

HEINE, B. 1997. Cognitive Foundations of Grammar. New York/Oxford: Oxford University Press.

HERSKOVITS, A. 1986. Language and Spatial Cognition: An Interdisciplinary Study of the Prepositions in English. Cambridge: Cambridge University Press.

HICKMANN, M. 2007. «Static and dynamic location in French: Developmental and cross-linguistic perspectives ». In M. Aurnague, M. Hickmann et L. Vieu (eds.). The Categorization of Spatial Entities in Language and Cognition, 205-231. Amsterdam: John Benjamins.

HICKMANN, M. et H. Hendriks. 2006. « Static and dynamic location in French and in English ». First Language. Volume 26, no. 1. 26: 103-135.

HILL, D. 1996. « Distinguishing the notion 'place' in an Oceanic language ». In M. Pütz et R. Dirven. The Construal of Space in Language and Thought, 307-328. Berlin : Mouton de Gruyter. HUSSERL, E. 1947 [2008]. Méditations Cartésiennes : Introduction à la Phénoménologie. Paris: VRIN. IBARRETXE-ANTUNANO, I. 2004. « Language typologies in our language use: the case of Basque motion events in adult oral narratives ». In Cognitive Linguistics. Volume 15 (3), 317-349. Copyright C. Mouton de Gruyter.

ISHIBASHI, M et A. Kopecka. 2011. « The (a)symmetry of Source and Goal ». Communication, Atelier « Trajectoire »: AFLiCo n 4, Lyon 2011.

JACKENDOFF, R. 1983. Semantics and Cognition. Cambridge, Mass.: MIT Press.

JACKENDOFF, R. 1993. Patterns in the Mind: Language and Human Nature. New York: Basic Books.

JACKENDOFF, R. 2002. Foundations of Language. Oxford: Oxford University Press.

JOHNSON, M. 1987. The Body in the Mind. The Bodily Basis of Meaning, Imagination and Reason. Chicago: University of Chicago Press.

JOHNSON, M. 1999. « Embodied Reason ». In Weiss, G. et F. H. Haber. Perspectives on Embodiment: The Intersections of Nature and Culture. New York/London: Routledge.

JOHNSON, M. 2007. The Meaning of the Body: Aesthetics of Human Understanding. Chicago/London: The University of Chicago Press.

JOHNSON, M., et T. Rohrer. 2007. « We are living creatures: Embodiment, American Pragmatism and the cognitive organism ». In T. Ziemke, J. Zlatev et R. M. Frank. (eds.). Body, Language and Mind, Volume 1: Embodiment, 17-54. Berlin: Mouton de Guyter.

JOSEPH, J. 2002. « The sources of the Sapir-Whorf hypothesis ». In Joseph, John, From Whitney to Chomsky. Essays in the History of American Linguistics, 71-105. Amsterdam/Philadelphia: John Benjamins.

KANT, E. 1835 [2006]. Critique de la Raison Pure. Paris : Flammarion.

KATZ, A.N., C. Cacciari, R. W. Gibbs, Jr., et M. Turner. 1998. Figurative Language and Thought. New York/Oxford: Oxford University Press. 
KERBRAT-ORECCHIONI, C. 2012. « Le contexte revisité ». Corela. RJC Cotexte, contexte, situation/ Numéros thématiques. Disponible sur : http://corela.revues.org/2627

KORZYBSKI, A. 1998. Une Carte n'est Pas le Territoire. Paris : Éditions de l'éclat.

LAKOFF, G., et M. Johnson. 1980. Metaphors We Live By. Chicago/London: The University of Chicago Press.

LAKOFF, G. 1987a. Women, Fire, and Dangerous Things. Chicago: The University of Chicago Press.

LAKOFF, G. 1987b. « Cognitive models and prototype theory ». In U. Neisser (ed.). Concepts and Conceptual Development: Ecological and Intellectual Factors in Categorization, 63-100. Cambridge: Cambridge University Press.

LAKOFF, G. et M. Johnson. 1999. Philosophy in the Flesh: The Embodied Mind and its Challenge to Western Thought. New York: Basic Books.

LANGACKER, R. 1976. « Semantic representations and the linguistic relativity hypothesis ». Foundations of Language, 14: 307-357.

LANGACKER, R. 1982. Space Grammar, Analysability, and the English Passive. in Language. 58, 1, Linguist Society of America: 22-80.

LANGACKER, R. 1987. Foundations of Cognitive Grammar, vol. 1. Stanford: Stanford University Press.

LANGACKER, R. 1991a. Foundations of Cognitive Grammar, vol. 2. Stanford: Stanford University Press.

LANGACKER, R. 1991b. Concept, Image, and Symbol: the Cognitive Basis of Grammar. Berlin/New York: Mouton de Gruyter.

LANGACKER, R. 1999. « Assessing the cognitive linguistic enterprise ». In T. Janssen et G. Redeker. Cognitive Linguistic Research: Foundations, Scope, and Methodology, 13-59. Berlin: Mouton de Gruyter.

LANGACKER, R. 1999. «A dynamic usage-based model ». In M. Barlow et S. Kemmer. (ed.). Usage Based Models of Language, 1-63. Stanford, California: CSLI Publications.

LANGACKER, R. 2000. Grammar and Conceptualization. Berlin/New York: Mouton de Gruyter.

LANGACKER, R. 2009. « Metonymic grammar ». In K. U. Panther, L.L. Thornburg, et A. Barcelona. Metonymy and Metaphor in Grammar, 45-71. Amsterdam/Philadelphia: John Benjamins Publishing Company.

LANGACKER, R. 2009. « Constructions and Constructional meaning ». In . Evans et S. Pourcel (eds.). New Directions in Cognitive Linguistics, 225-267. Amsterdam/Philadelphia: John Benjamins Publishing Company.

LEDERER, A., H.; Gleitman et L. Gleitman. 1995. « Verbs of a feather flock together: semantic information in the structure of maternal speech ». In M., Tomasello, et W. E. Merriman. Beyond Names for Things: Young Children's Acquisition of Verbs, 277-297. Hillsdale : Lawrence Erlbaum Associates.

LEMMENS, M. 2004. « Motion and location: toward a cognitive typology ». In Girard, G. (éd.) Parcours Linguistique. Domaine Anglais, 223-244. [Travaux $122 \mathrm{du}$ Cierec]. Publications de l'Université de St Étienne.

MANDLER, J. M. 2005. « How to build a baby: III. Image schemas and the transition to verbal thought ». In B. Hampe (ed.). From Perception to Meaning: Image Schemas in Cognitive Linguistics, 137-163. Berlin: Mouton de Gruyter.

MANDLER, J. M. 2010. " The spatial foundations of the conceptual system ». In D. Casasanto et al. Language and cognition. vol. 2. Number 1, 21-44. Berlin/New York: Mouton de Gruyter. 
MATSUMOTO, Y. 1996. « How Abstract is Subjective Motion? A Comparison of Coverage Path Expressions and Acces Path Expressions ». In A. Goldberg. Conceptual Structures, Discourse and language, 359-373. CSLI Publications.

MATSUMOTO, Y. 2011. « Varieties of Path Expressions within and across Languages ». Communication, AFLiCo n 4, Lyon, Mai 2011.

Mc NEILL, D. 1992. Hand and Mind: What Gestures Reveal about Thought. Chicago and London: The University of Chicago Press.

MILLER, G. A. et P. N. Johnson-Laird. 1976. Language and Perception. Harvard: Harvard University Press.

PIAGET, J. 1950. The Psychology of Intelligence. London/New York: Routledge.

PIAGET, J. 1977. La Construction du Réel chez L'Enfant. Neuchâtel : Delachaux et Niestlé.

PINKER, S. 1997. How the Mind Works. London. Penguin.

PODHORODECKA, J. 2007. Evaluative Metaphor: Extended Meanings of English Motion Verbs. Kraków: Wydawnictwo Naukowe Akademii Pedagogicznej.

POURCEL, S. 2009. « Motion scenarios in cognitive processes ». In V. Evans et S. Pourcel (ed.). New Directions in Cognitive Linguistics, 371-391. Amsterdam/Philadelphia: John Benjamins Publishing Company.

ROHLFING, K. J. 2011. « Meaning in the objects ». In J. Meibauer, et M.Steinbach (eds.).

Experimental Pragmatics/Semantics, 151-176. Amsterdam/Philadelphia: John Benjamins Publishing Company.

SAEED, J. I. 1997. Semantics. Malden/Oxford: Blackwell Publishing.

SIDNELL, J. 2009. « Deixis ». In Verschueren, J., and J.-O. Östman. Key Notions for Pragmatics, 114-138. Amsterdam/Philadelphia: John Benjamins Publishing Company.

SLOBIN, Dan I. 1977. « Language change in childhood and in history ». In J. Macnamara (ed.). Language, Learning and Thought. London: Academic Press.

SLOBIN, Dan I. 1991. « Learning to think for speaking: Native language, cognition, and rhetorical style ». Pragmatics 1: 7-26.

SLOBIN, Dan I. 1996a. « From 'thought to language' to 'thinking for speaking. ». In J.J. Gumperz and Stephen C. Levinson (eds). Rethinking Linguistic Relativity, 70-96. Cambridge: Cambridge University Press.

SLOBIN, Dan I. 1996b. « Two ways to travel: Verbs of motion in English and Spanish ». In Grammatical Constructions: Their Form and Meaning, 195-217. In M. Shibatani and S.A. Thompson (eds). Oxford: Oxford University Press.

SLOBIN, Dan I. 1997. « Mind, Code, and Text ». In J. Bybee, J. Haiman et S. A. Thompson (eds.). Essays on Language Function and Language Type, 437-67. Amsterdam: John Benjamins.

SLOBIN, Dan I. 2003a. « Language and Thought Online: cognitive consequences of linguistic relativity ». In D. Gentner et S. Goldin-Meadow (eds). Language in Mind: Advances in the Study of Language and Thought, 157-192. Cambridge: MIT Press.

SLOBIN, Dan I. 2003b. « Relations between Paths of Motion and Paths of Vision: A Crosslinguistic and Developmental Exploration ». In V. M. Gathercole (ed.), Routes to Language: Studies in honor of Melissa Bowerman, 197-222. A New York/London: Psychology Press. 
SLOBIN, Dan I. 2004. " The many ways to search for a frog: Linguistic typology and the expression of motion events ». In S. Strömqvist et L. Verhoeven (eds.) Relating Events in Narrative: vol. 2. Typological and Contextual Perspectives, 219-257. Mahwah, N. J: Lawrence Erlbaum Associates Publishers.

SONESSON, G. 2007. «From the meaning of embodiment to the embodiment of meaning: A study in phenomenological semiotics ». In T. Ziemke, J. Zlatev et R. M. Frank. (eds.). Body, Language and Mind, Volume 1: Embodiment, 85-127. Berlin: Mouton de Guyter.

STEEN, G. J. 2007. Finding Metaphor in Grammar and Usage. Amsterdam/Philadelphia: John Benjamins Publishing Company.

SVOROU, S. 1994. The Grammar of Space. Amsterdam/Philadelphia: John Benjamins Publishing Company.

TALMY, L. 1978. « Figure and Ground in complex sentences ». In Joseph H. Greenberg (ed), Universals of Human Language (Vol. 4), Standford, Calif.: Standford University Press. Repris dans Towards a Cognitive Semantics. vol. 1, 2000. MIT Press.

TALMY, L. 1983. « How language structures space ». In Herbert L. Pick, Jr., et Linda P. Acredolo (eds). Spatial Orientation: Theory, research, and application, 225-282. New York: Plenum Press. Repris dans Towards a Cognitive Semantics, vol. 1, 2000. MIT Press.

TALMY, L. 1985. « Lexicalization patterns: Semantic structure in lexical forms ». In Tim Shopen (ed.). Language Typology and Syntactic Description. vol. 3: Grammatical Categories and the Lexicon, 57-149. Cambridge: Cambridge University Press.

TALMY, L. 2000a. Toward a cognitive semantics: Concept Structuring Systems. Volume.1. Cambridge, Mass.: MIT Press.

TALMY, L. 2000b. Toward a cognitive semantics: Typology and Process in Concept Structuring. Volume.2. Cambridge, Mass.: MIT Press.

TALMY, L. 2005. «The fundamental system of spatial schemas in language ». In B. Hampe. From Perception to Meaning: Image Schemas in Cognitive Linguistics, 199-234. Berlin: Mouton de Gruyter.

TAYLOR, J.R. 2002. Cognitive Grammar. Oxford: Oxford University Press.

TURNER, M. 1996. The Literary Mind. Oxford/New York: Oxford University Press.

VANDELOISE, C. 2006. De la Distribution à la Cognition. Paris : L'Harmattan

VANDELOISE, C. 2007b. « Le verbe aller : l'affranchissement du contexte d'énonciation immédiat ». Journal of French Language Studies, 17(3) :343-359.

VICTORRI, B. 2010. « Le localisme à l'épreuve du verbe aller ». vol. 8. Corela. Disponible sur : http://corela.revues.org/1009

VYGOTSKY, L. 1962. Thought and Language. Cambridge, Massachusetts: MIT Press.

\section{NOTES}

1. On entend par « conceptualisation » l'action d'attribuer une unité ou une combinaison d'unités lexicales et / ou grammaticales, pour décrire un état de fait. La conceptualisation, telle qu'elle est examinée dans cette étude, relève de la description et de la catégorisation (Croft, 1991 : 101-103). 2. Plusieurs traductions sont proposées pour le terme image schema; nous proposons dans cette étude celle de "schème-image ". 
3. Dans le présent travail, on considère une «entité » comme topologiquement individuable et stable (Col, 2010 : § 32).

4. Pour une définition récente du schème-image du chemin, voir Barnabé, 2010.

5. Compte tenu du caractère abstrait $d u$ schème $d u$ chemin, l'expression langagière $d u$ « chemin » a fait l'objet de plusieurs approches théoriques qui présentent des traits similaires. On compte, parmi elles, celles de Lakoff (1987), Matsumoto (1996), Slobin (1996a, 1996b, 1991, 1997, 2003, 2004, 2009), Talmy (2000a, 2000b), Langacker (1991, 1999, 2000, 2010), Jackendoff (1983) et Taylor (2002), pour ne citer que les principales sources.

6. L'ICM peut aussi être métaphorique, métonymique, ou symbolique (symbolic). En LC, on doit le concept d'ICM au frames de Fillmore (1985), à la théorie de la métaphore et de la métonymie de Lakoff et Johnson (1980), à la grammaire cognitive de Langacker (1982) et à la théorie des espaces mentaux de Fauconnier (1994).

7. BLOCKED PATH: "Infants as young as two months expect that a rolling ball will not go through an object in front of it" (Mandler, $2010: 29$ ).

8. "Perceptual meaning analysis is an attentive process that analyses perceptual displays and recodes them into a reduced form that makes conscious thought possible" (Mandler, $2005: 140$ ).

9. "Satellite-framed languages express the Path in a satellite and the verb expresses a Co-event, usually Manner or Cause" (Lemmens, $2004: 5$ ).

10. Les cinq premiers exemples de la présente analyse sont extraits du recueil de données utilisé par A. Barnabé dans sa thèse de doctorat (Barnabé, 2012).

11. Les éléments figure et ground (Talmy, 2000a, 2000b) correspondent aux éléments trajector/ landmark (Langacker, 1991a, 1991b, 1999; Fauconnier \& Turner, 2002), et à la cible et au site (Vandeloise, 2006). Dans la présente étude, on emploiera les termes «figure » et «fond » pour désigner ces deux entités.

12. "The term 'deixis' from the Greek for pointing, refers to a particular way in which the interpretation of certain linguistic expressions ('deictics' or 'indexical') is dependent on the context in which they are produced or interpreted" (Sidnell, $2009: 114$ ).

13. Talmy commente le statut de la deixis: "The Deictic is thus just a special choice of Vector, Conformation, and Ground, not a semantically distinct factor, but its recurrence across languages earns its structural status" (Talmy, 2000b : 138).

14. À partir d'expériences réalisées avec de jeunes anglophones, K. J. Rohlfing note toutefois que si les jeunes individus intègrent le sens de on relativement tôt, celui de under est maîtrisé de manière très approximative à l'âge de deux ans.

15. Dans la même veine, E. Andersen a effectué des analyses relatives à l'identification des parties du corps par le jeune sujet parlant, afin de déterminer la nuance de localisation entre la partie supérieure et la partie inférieure du corps: upper body versus lower body (Andersen, 1978: 335-368).

16. Les langues à cadrage verbal concentrent l'expression du mouvement et du chemin dans l'unité verbale (par ex. « Il entra dans la cuisine »).

17. D'une manière identique, Slobin (1977) réalise plusieurs études comparatives, relatives à l'acquisition de l'ordre des mots chez de jeunes sujets exprimant les phénomènes de mouvement et de localisation, à partir d'une gamme de langues plus large que celle de l'étude de Tomasello.

18. Pour une schématisation de la localisation anglophone et coréenne, voir Tomasello (2003: $64)$.

19. "Satellite-framed languages express the Path in a satellite and the verb expresses a Co-event, usually Manner or Cause" (Lemmens, $2004: 5$ ).

20. La typologie de Talmy est S-framed / V-framed en matière de constructions, non en matière de langues (pour lesquelles il ajoute les split systems et parallel systems). La typologie oppose les langues à cadrage verbal (espagnol, français, italien, turque, hébreu, japonais, langues sémitiques, tamil, polynésien, bantou, quelques branches de la langue Maya, Nez Percé, langue 
Caddo), aux langues à satellites (anglais, allemand, néerlandais, russe, mandarin, chinois, langues finno-ougriennes, ojibwa, warlpiri).

21. Par acception spatiale, nous entendons un sens concret qui représente une réalité tangible.

22. Ils apparaissent entre parenthèses, tels qu'ils sont signalés dans cocA.

23. Dixon (1991: 7).

24. On fait référence au phénomène du double framing, qui fait écho au split system (Talmy, 2000b). Ce phénomène fait référence à des langues à cadrage verbal qui affichent des constructions réservées aux langues à satellites et réciproquement.

25. Pour plus de détails sur l'ambiguité catégorielle des satellites, voir Matsumoto (2011) et Grinevald et al. (2011).

26. Cette caractéristique s'applique principalement en atsugewi et en russe (Ibid. : 115-122).

27. Les enquêtes de Haviland (1986), explique Givón (1989), démontrent la réalité de la situation réciproque invoquée par Miller-Johnson-Laird (1976), en se fiant à la culture aborigène Guugu Yimidhirr, dont les seuls repères spatiaux sont les points cardinaux.

28. «Cotexte : matériel sémiotique qui précède et suit la séquence soumise à l'analyse » (KerbratOrecchioni, 2012 : § 96).

29. Nous traduisons littéralement construed restrictively (Langacker, 1991b : 96).

30. Nous employons ici la "forme perfective" en un sens particulier qui est celui de Langacker pour qui une forme progressive s'applique à un verbe porteur d'un sens qu'il appelle "perfectif".

31. Nous incluons les concepts mouvement / localisation dans un domaine plus large, celui de la vélocité. On se référera au mouvement par la «vélocité 1 » et au stationnement (ou localisation) par la « vélocité 0 ».

32. L'Agonist fait partie des entités qui interagissent dans la catégorie sémantique désignée dynamique des forces (force dynamics) par Talmy. Talmy représente schématiquement l'Agonist comme l'entité exerçant un type de force, qui s'oppose à l'Antagonist, i.e. celui qui y résiste (Talmy, 2000a : 415).

33. On précise ici que cette paraphrase s'applique à went under. Notre théorie se sépare donc de celle de Jackendoff, pour qui cette paraphrase correspond à l'un des sens de under. Ainsi, nous choisissons de considérer went under, non under, comme potentiellement ambigu.

34. A partir du classement de Langacker qui divise les prépositions suivant la terminologie «1way " et "2-way" prepositions, la troisième catégorie d'unités prépositionnelles est désignée par "3-way prepositions ", par cohérence terminologique. Cette catégorie représente la complexité constructionnelle des prépositions qu'elle inclut. (Talmy, 2000a : 312-313).

35. Nous conservons l'adjectif employé par Slobin dans sa description des "elaborated complex paths": "He still wandered on, out of the little high valley, over its edge, and down the slopes beyond" (Slobin, 1997 : 439).

36. La préposition with n'est pas prise en compte dans la mesure où elle ne spécifie aucune direction.

37. On note que la structure syntaxique diffère en (34) et (35) (qui pourrait s'analyser comme contenant une ellipse, par "gapping", et donc contenant deux propositions). 


\section{RÉSUMÉS}

La linguistique cognitive conçoit l'organisation du lexique et des constructions comme les traces de la conceptualisation de l'expérience du monde par le sujet parlant. Elle considère les structures langagières comme le reflet de structures conceptuelles sous-jacentes. Les schèmesimages font partie de ces structures. Les schèmes-images sont construits et abstraits à partir de l'expérience incorporée et socialement située du monde, ce qui leur confère à la fois une assise culturelle et sensori-motrice. Ces schèmes dits préconceptuels correspondent à des structures gestaltiques qui facilitent l'appréhension du sujet aux dimensions de son expérience. L'objectif du présent article consiste à retracer les conditions d'élaboration du schème du chemin en tenant compte de la dimension kinesthésique de son développement. La quête épistémologique du concept-même de «schème-image " permettra d'aborder avec plus de justesse la structuration linguistique de l'expérience vécue du schème du chemin, dont ressort la spatialité comme guide mais surtout comme contrainte et base de conceptualisations multiples de l'organisme cognitif. Par ailleurs, cette étude tentera d'assigner une structuration prépositionnelle au schème-image du chemin à partir de micro-analyses d'exemples issus d'un recueil de 500 occurrences.

Cognitive linguistics posits that linguistic structures are tied to conceptual structures. Image schemas belong to these structures. Schemas are shaped by socio-physical experience, and accordingly they have a firm cultural and sensor-motor basis. The present study aims at accounting for the development of the PATH schema on the basis of its kinesthetic origin. The epistemic investigation of the mere concept of "image-schema" will make it easier to focus on the linguistic structure of the PATH schema and of its enacted experience. The most fundamental concept arising from this image-schema is the experience of space, which represents a guide as well as a constraint for the conceptualizer. It also marks the basis of multiple conceptualisations of the cognitive being. Moreover, in this study, we will try to assign a prepositional structure to the PATH schema on the basis of micro-analyses of examples extracted from a corpus-based analysis composed of 500 occurrences.

\section{INDEX}

Mots-clés : schème-image du chemin, conceptualisation, structuration syntaxique, mouvement, localisation, prépositions

Keywords : path image schema, conceptualization, syntaxic structuration, motion, localization, preposition

\section{AUTEUR}

\section{AURÉLIE BARNABÉ}

Université Blaise-Pascal, Clermont-Ferrand 